\title{
RANK 2 SYMMETRIC HYPERBOLIC KAC-MOODY ALGEBRAS
}

\author{
SEOK-JIN KANG* AND DUNCAN J. MELVILLE
}

\section{Introduction}

Affine Kac-Moody algebras represent a well-trodden and well-understood littoral beyond which stretches the vast, chaotic, and poorly-understood ocean of indefinite Kac-Moody algebras. The simplest indefinite Kac-Moody algebras are the rank 2 Kac-Moody algebras $\mathfrak{g}(a)(a \geq 3)$ with symmetric Cartan matrix $\left(\begin{array}{cc}2 & -a \\ -a & 2\end{array}\right)$, which form part of the class known as hyperbolic Kac-Moody algebras. In this paper, we probe deeply into the structure of those algebras $g(a)$, the $e$. coli of indefinite Kac-Moody algebras. Using Berman-Moody's formula $([\mathrm{BM}])$, we derive a purely combinatorial closed form formula for the root multiplicities of the algebra $\mathrm{g}(a)$, and illustrate some of the rich relationships that exist among root multiplicities, both within a single algebra and between different algebras in the class. We also give an explicit description of the root system of the algebra $g(a)$. As a by-product, we obtain a simple algorithm to find the integral points on certain hyperbolas.

For alternative approaches to the analysis of rank 2 hyperbolic Kac-Moody algebras, the reader should see [BKM, Section 4], which constructs them as $8 l_{2}$-modules, and [LM], which shows that the root systems of these algebras coincide with those of quasi-regular cusps on Hilbert modular surfaces defined over certain real quadratic fields.

The structure of the paper is as follows. In Section 1, we introduce Berman-Moody's root multiplicity formula for general symmetrizable Kac-Moody algebras. In Section 2, we specialize to the rank 2 hyperbolic Kac-Moody algebras

Received July 22, 1994.

* Supported in part by Basic Science Research Institute Probram, Ministry of Education of Korea, BSRI-94-1414 and GARC-KOSEF at Seoul National University, Korea. 
$g(a)$ and develop a purely combinatorial formula (2.14) for the root multiplicities. We show how, in many cases, one can obtain the multiplicity of a root in one algebra from the multiplicity of the same root in a different algebra, which may have much simpler calculations. Section 3 extends this notion considerably by proving a stability theorem on the root multiplicities of $\mathfrak{g}(a)$ as $a$ increases and explaining the connections between the algebras $g(a)$ and the free Lie algebra of rank 2. The stability theorem holds for arbitrary symmetrizable Kac-Moody algebras.

In Section 4, we consider the root system of the algebra $g(a)$. Recalling that real roots and imaginary roots correspond to the integral points on the hyperbolas: $x^{2}-a x y+y^{2}=k(k \in \mathbf{Z}, k \leq 1)$, we show how all the roots of a given length are Weyl-conjugate to roots in a small and easily defined region. Thus we can easily list all the roots, with multiplicity, by use of some simple recurrence relations. This procedure finds all the integral points on these hyperbolas far more easily than the traditional number-theoretic algorithm.

In Section 5, we analyze some of the monotonic and symmetric relationships between the root multiplicities of the algebra $g(a)$ for a fixed value of $a$. We raise some questions concerning possible relationships among the root multiplicities for given algebras. We conjecture that the multiplicities of roos of a given height $t$ in crease monotonically to a maximum at $(m, m)$ for $t$ even, and $(m-1, m+1)$ for $t$ odd. The paper closes with a collection of tables illustrating the main results and the conjecture.

\section{Berman-Moody's formula}

We first recall some of basic definitions in Kac-Moody theory and BermanMoody's formula. Let $I$ be an index set. A matrix $A=\left(a_{i j}\right)_{i, j \in I}$ is called a generalized Cartan matrix if it satisfies: (i) $a_{i i}=2$ for all $i \in I$, (ii) $a_{i j} \in \mathbf{Z}_{\leq 0}$ for $i \neq j$, (iii) $a_{i j}=0$ if and only if $a_{j i}=0$. In this paper, we assume that $A$ is symmetrizable, i.e., there is an invertible diagonal matrix $D$ such that $D A$ is symmetric. A realization of $A$ is a triple $\left(\mathfrak{h}, \Pi, \Pi^{\vee}\right)$, where $\mathfrak{h}$ is a complex vector space of dimen$\operatorname{sion} 2|I|-\operatorname{rank} A, \Pi=\left\{a_{i} \mid i \in I\right\}$ and $\Pi^{\vee}=\left\{h_{i} \mid i \in I\right\}$ are linearly independent subsets of $\mathfrak{h}^{*}$ and $\mathfrak{h}$, respectively, satisfying $a_{j}\left(h_{i}\right)=a_{i j}$ for $i, j \in I$.

Definition 1.1. The Kac-Moody algebra $\mathfrak{g}=\mathfrak{g}(A)$ with Cartan matrix $A$ is the Lie algebra generated by the elements $e_{i}, f_{i}(i \in I)$ and $\mathfrak{h}$ with the following defin- 
ing relations:

$$
\begin{aligned}
& {\left[h, h^{\prime}\right]=0 \text { for } h, h^{\prime} \in \mathfrak{h},} \\
& {\left[h, e_{j}\right]=a_{j}(h) e_{j},\left[h, f_{j}\right]=-a_{j}(h) f_{j} \text { for } j \in I,} \\
& {\left[e_{j}, f_{i}\right]=\delta_{i j} h_{i} \text { for } i, j \in I,} \\
& \left(a d e_{i}\right)^{1-a_{i j}}\left(e_{j}\right)=\left(a d f_{i}\right)^{1-a_{i j}}\left(f_{j}\right)=0 \text { for } i \neq j .
\end{aligned}
$$

The elements of $\Pi$ (resp. $\Pi^{\vee}$ ) are called the simple roots (resp. simple coroots) of $\mathrm{g}$. For each $i \in I$, let $r_{i} \in$ Aut $\left(\mathfrak{h}^{*}\right)$ be the simple reflection on $\mathfrak{h}^{*}$ defined by $r_{i}(\lambda)=$ $\lambda-\lambda\left(h_{i}\right) \alpha_{i}$. The subgroup $W$ of $G L\left(\mathfrak{h}^{*}\right)$ generated by the $r_{i}^{\prime}$ s $(i \in I)$ is called the Weyl group of $\mathfrak{g}$.

Let $Q=\bigoplus_{i \in I} \mathbf{Z} a_{i}, Q_{+}=\bigoplus_{i \in I} \mathbf{Z}_{\geq 0} a_{i}$, and $Q_{-}=-Q_{+}$. We define a partial ordering $\geq$ on $\mathfrak{h}^{*}$ by $\lambda \geq \mu$ if and only if $\lambda-\mu \in Q_{+}$. The Kac-Moody algebra $\mathfrak{g}=\mathrm{g}(A)$ has the root space decomposition $\mathrm{g}=\bigoplus_{\alpha \in Q} \mathrm{~g}_{\alpha}$, where $\mathfrak{g}_{\alpha}=\{x \in$ $\mathrm{g} \mid[h, x]=\alpha(h) x$ for $h \in \mathfrak{h}\}$ is the $\alpha$-root space. An element $\alpha \in Q$ is called a root if $a \neq 0$ and $\mathfrak{g}_{\alpha} \neq 0$. The number $\operatorname{mult}(\alpha):=\operatorname{dimg}_{\alpha}$ is called the multiplicity of the root $\alpha$. A root $\alpha>0$ (resp. $\alpha<0$ ) is called positive (resp. negative). It is known that all the roots are either positive or negative. We denote by $\Delta, \Delta^{+}$, and $\Delta^{-}$the set of all roots, and negative roots, respectively. For $\alpha=\sum_{i \in I} k_{i} \alpha_{i} \in Q$, the number $\operatorname{ht}(\alpha):=\sum_{i \in I} k_{i}$ is called the height of $\alpha$.

A $\mathfrak{g}$-module $V$ is $\mathfrak{h}$-diagonalizable if $V=\bigoplus_{\lambda \in \mathfrak{h}} * V_{\lambda}$, where $V_{\lambda}=\{v \in V \mid h \cdot v$ $=\lambda(h) v$ for all $h \in \mathfrak{h}\}$ is the $\lambda$-weight space. If $V_{\lambda} \neq 0$, then $\lambda$ is called a weight of $V$. The number $\operatorname{mult}_{V}(\lambda):=\operatorname{dim} V_{\lambda}$ is called the multiplicity of $\lambda$. When all the weight spaces are finite-dimensional, we define the character of $V$ to be

$$
\operatorname{ch} V=\sum_{\lambda \in \mathfrak{h}^{*}}\left(\operatorname{dim} V_{\lambda}\right) e^{\lambda} .
$$

An $\mathfrak{h}$-diagonalizable module $V$ is integrable if all the $f_{i}(i \in I)$ are locally nilpotent on $V$. A g-module $V$ is called a highest weight module with highest weight $\lambda \in \mathfrak{h}^{*}$ if there is nonzero vector $v_{0} \in V$ such that (i) $e_{i} \cdot v_{0}=0$ for all $i \in I$, (ii) $h \cdot v_{0}=$ $\lambda(h) v_{0}$ for all $h \in \mathfrak{h}$, (iii) $U(\mathfrak{g}) \cdot v_{0}=V$. The vector $v_{0}$ is called a highest weight vector. We denote by $V(\lambda)$ the irreducible highest weight module over $\mathfrak{g}$.

Let $S \subset I$ and $\mathrm{g}_{S}=\mathrm{g}\left(A_{S}\right)$ be the Kac-Moody algebra with Cartan matrix $A_{S}=\left(a_{i j}\right)_{i, j \in S}$. We denote by $\Delta_{S}, \Delta_{S}^{ \pm}$, and $W_{S}$ the set of roots, the set of positive (resp. negative) roots, and the Weyl group of $\mathfrak{g}_{s}$, respectively. Let $\Delta^{ \pm}(S)=\Delta^{ \pm} \backslash \Delta_{S}^{ \pm}$, and $W(S)=\left\{w \in W \mid \Phi_{w} \subset \Delta^{+}(S)\right\}$, where $\Phi_{w}=\left\{\alpha \in \Delta^{+} \mid w^{-1}(\alpha)<0\right\}$. We also define $\mathfrak{g}_{0}^{(S)}=\mathfrak{g}_{S}+\mathfrak{h}$, and $\mathfrak{g}_{ \pm}^{(S)}=\bigoplus_{\alpha \in \Delta^{ \pm}(S)} \mathfrak{g}_{\alpha}$. Then we have the triangular de- 
composition:

$$
\mathfrak{g}=\mathfrak{g}_{-}^{(S)} \oplus \mathfrak{g}_{0}^{(S)} \oplus \mathfrak{g}_{+}^{(S)}
$$

Let $\mathbf{C}$ be the trivial $\mathfrak{g}$-module. The homology modules $H_{k}\left(\mathfrak{g}_{-}^{(S)}, \mathbf{C}\right)$ are obtained from the $\mathfrak{g}_{0}^{(S)}$-module complex

$$
\begin{aligned}
\cdots \rightarrow \Lambda^{k}\left(\mathrm{~g}_{-}^{(S)}\right) \stackrel{d_{k}}{\rightarrow} & \Lambda^{k-1}\left(\mathrm{~g}_{-}^{(S)}\right) \rightarrow \cdots \\
& \rightarrow \Lambda^{1}\left(\mathrm{~g}_{-}^{(S)}\right) \stackrel{d_{1}}{\rightarrow} \Lambda^{0}\left(\mathrm{~g}_{-}^{(S)}\right) \stackrel{d_{0}}{\rightarrow} \mathbf{C} \rightarrow 0,
\end{aligned}
$$

with the differentials $d_{k}: \Lambda^{k}\left(\mathfrak{g}_{-}^{(S)}\right) \rightarrow \Lambda^{k-1}\left(\mathfrak{g}_{-}^{(S)}\right)$ defined by

$$
d_{k}\left(x_{1} \wedge \cdots \wedge x_{k}\right)=\sum_{s<t}(-1)^{s+t}\left(\left[x_{s}, x_{t}\right] \wedge x_{1} \wedge \cdots \wedge \widehat{x_{s}} \wedge \cdots \wedge \widehat{x_{t}} \wedge \cdots \wedge x_{k}\right)
$$

for $x_{i} \in \mathfrak{g}_{-}^{(S)}$. For simplicity, we write $H_{k}\left(\mathfrak{g}_{-}^{(S)}\right)$ for $H_{k}\left(\mathfrak{g}_{-}^{(S)}, \mathbf{C}\right)$. The $\mathfrak{g}_{0}^{(S)}$-module structure of the homology modules $H_{k}\left(\mathfrak{g}_{-}^{(S)}\right)$ is determined by the following formula known as Kostant's formula.

Proposition 1.2 ([GL], [Li]).

$$
H_{k}\left(\mathfrak{g}_{-}^{(S)}\right) \cong \underset{\substack{w \in W(S) \\ l(w)=k}}{\bigoplus} V_{S}(w \rho-\rho),
$$

where $V_{S}(\lambda)$ denotes the irreducible highest weight $\mathrm{g}_{0}^{(S)}$-module with highest weight $\lambda$, and $\rho$ is an element of $\mathfrak{h}^{*}$ satisfying $\rho\left(h_{i}\right)=1$ for all $i \in I$.

We now recall the root multiplicity formula for $\mathfrak{g}$ obtained in [Ka2]. Applying the Euler-Poincaré principle to (1.4) yields:

$$
\sum_{k=0}^{\infty}(-1)^{k} \operatorname{ch} \Lambda^{k}\left(g_{-}^{(S)}\right)=\sum_{k=0}^{\infty}(-1)^{k} \operatorname{ch} H_{k}\left(g_{-}^{(S)}\right)
$$

Let

$$
\begin{aligned}
H & =\sum_{k=0}^{\infty}(-1)^{k+1} H_{k}\left(\mathfrak{g}_{-}^{(S)}\right)=\sum_{k=0}^{\infty}(-1)^{k+1} \sum_{\substack{w \in W(S) \\
l(w)=k}} V_{S}(w \rho-\rho) \\
& =\sum_{\substack{w \in W(S) \\
l(w) \geq 1}}(-1)^{l(w)+1} V_{S}(w \rho-\rho),
\end{aligned}
$$

an alternating direct sum of $g_{0}^{(S)}$-modules. For $\tau \in Q_{-}$, we define the dimension of the $\tau$-weight space of $H$ to be

$$
\operatorname{dim} H_{\tau}=\sum_{k=1}^{\infty}(-1)^{k+1} \operatorname{dim} H_{k}\left(\mathrm{~g}_{-}^{(S)}\right)_{\tau} .
$$


Let $P(H)=\left\{\tau \in Q_{-} \mid \operatorname{dim} H_{\tau} \neq 0\right\}$ and let $\left\{\tau_{\imath} \mid i \geq 1\right\}$ be the enumeration of $P(H)$ given by the height and lexicographical ordering. For $\tau \in Q_{-}$, we define a set

$$
T(\tau)=\left\{(n)=\left(n_{i}\right)_{i \geq 1} \mid n_{i} \in \mathbf{Z}_{\geq 0}, \sum n_{i} \tau_{i}=\tau\right\}
$$

and a function

$$
B(\tau)=\sum_{(n) \in T(\tau)} \frac{\left(\sum n_{i}-1\right) !}{\Pi\left(n_{i} !\right)} \Pi\left(\operatorname{dim} H_{\tau_{i}}\right)^{n_{i}}
$$

Then we have:

Theorem 1.3 ([Ka2]). Let $\alpha$ be a root in $\Delta^{-}(S)$. Then

$$
\operatorname{dimg}_{\alpha}=\sum_{\tau \mid \alpha} \mu\left(\frac{\alpha}{\tau}\right) \frac{\tau}{\alpha} B(\tau)
$$

where $\mu$ is the classical Möbius function and $\tau \mid \alpha$ if $\alpha=k \tau$ for some positive integer $k$, in which case $\frac{\alpha}{\tau}=k$ and $\frac{\tau}{\alpha}=\frac{1}{k}$.

If $S=\phi$, then we have $W(S)=W$, and

$$
H=\sum_{\substack{w \in W \\ l(w) \geq 1}}(-1)^{l(w)+1} \mathbf{C}_{w \rho-\rho} .
$$

Hence $P(H)=\{w \rho-\rho \mid w \in W\}$. Let $\left\{\tau_{i}=w_{i} \rho-\rho \mid i \geq 1\right\}$ be the enumeration of the set $P(H)$ given by the height and lexicographical ordering. Then we have

$$
\operatorname{dim} H_{\tau_{i}}=\sum_{\substack{w \in W \\ l(w) \geq 1}}(-1)^{l(w)+1} \delta_{w \rho-\rho, \tau_{i}}=(-1)^{l\left(w_{i}\right)+1} .
$$

Therefore the formula (1.11) reduces to Berman-Moody's formula:

COROLlary $1.4([\mathrm{BM}])$.

$$
\operatorname{dim}_{\alpha}=\sum_{\tau \mid \alpha} \mu\left(\frac{\alpha}{\tau}\right) \frac{\tau}{\alpha} \sum_{(n) \in T(\tau)} \frac{\left(\sum n_{i}-1\right) !}{\Pi\left(n_{i} !\right)} \Pi\left((-1)^{l\left(w_{i}\right)+1}\right)^{n_{i}} .
$$

\section{The hyperbolic Kac-Moody algebras $g(a)$}

In this section, we study the structure of rank 2 hyperbolic Kac-Moody 
algebras $\mathfrak{g}(a)$ with symmetric Cartan matrix $\left(\begin{array}{cc}2 & -a \\ -a & 2\end{array}\right)$ using Berman-Moody's formula. Let $I=\{0,1\}$ be the index set for the simple roots of $\mathrm{g}(a)$, and take $S=$ $\phi$. Then $\mathfrak{g}(a)_{0}^{(S)}=\mathfrak{h}=\mathbf{C} h_{0} \oplus \mathbf{C} h_{1}$, the Cartan subalgebra, and $\Delta^{-}(S)=\Delta^{-}$. Moreover, $W(S)=W$, the full Weyl group, and we have an explicit description of $W$ :

$$
W=\left\{1, r_{0}\left(r_{1} r_{0}\right)^{j}, r_{1}\left(r_{0} r_{1}\right)^{j},\left(r_{0} r_{1}\right)^{j+1},\left(r_{1} r_{0}\right)^{j+1} \mid j \geq 0\right\}
$$

Hence, by (1.12), we have

$$
\begin{aligned}
H & =\sum_{\substack{w \in W \\
l(w) \geq 1}}(-1)^{l(w)+1} \mathbf{C}_{w \rho-\rho} \\
& =\frac{\sum_{j \geq 0}\left(\mathbf{C}_{r_{0}\left(r_{1} r_{0}\right)^{j} \rho-\rho} \oplus \mathbf{C}_{r_{1}\left(r_{0} r_{1}\right)^{j} \rho-\rho}\right)}{\sum_{j \geq 0}\left(\mathbf{C}_{\left(r_{0} r_{1}\right)^{j+1} \rho-\rho} \oplus \mathbf{C}_{\left(r_{1} r_{0}\right)^{j+1} \rho-\rho}\right)},
\end{aligned}
$$

where we let $A / B$ denote $A \ominus B$.

We introduce a sequence $\left\{A_{n}\right\}_{n \geq 0}$ defined as follows:

$$
\begin{aligned}
A_{0} & =0, \quad A_{1}=1, \\
A_{n+2} & =a A_{n+1}-A_{n}+1 \text { for } n \geq 0 .
\end{aligned}
$$

When we want to emphasize that the sequence $\left\{A_{n}\right\}_{n \geq 0}$ depends on $a$, we will write $\left\{A_{n}(a)\right\}_{n \geq 0}$. Since the sequence $\left\{A_{n}\right\}_{n \geq 0}$ will play a crucial role in writing the root multiplicity formula for $g(a)$, we investigate some of the basic properties of the sequence $\left\{A_{n}\right\}_{n \geq 0}$.

The first few terms of the sequence $\left\{A_{n}\right\}_{n \geq 0}$ are

$$
\begin{aligned}
& A_{0}=0, A_{1}=1, A_{2}=1+a, A_{3}=a+a^{2}, \\
& A_{4}=-a+a^{2}+a^{3}, \cdots .
\end{aligned}
$$

When $a=3$, we have $A_{n}=F_{2 n}-1$, where $\left\{F_{n}\right\}_{n \geq 0}$ is the Fibonacci sequence defined by

$$
F_{0}=F_{1}=1, F_{n+2}=F_{n+1}+F_{n}(n \geq 0) .
$$

To see this, let $A_{n}^{\prime}=A_{n}+1$. Then $A_{0}^{\prime}=1, A_{1}^{\prime}=2$, and for $n \geq 0$

$$
\begin{aligned}
A_{n+2}^{\prime} & =A_{n+2}+1=3 A_{n+1}-A_{n}+2 \\
& =3\left(A_{n+1}+1\right)-\left(A_{n}+1\right)=3 A_{n+1}^{\prime}-A_{n}^{\prime} .
\end{aligned}
$$

On the other hand, (2.4) yields 


$$
\begin{aligned}
F_{2 n+4} & =F_{2 n+3}+F_{2 n+2}=2 F_{2 n+2}+F_{2 n+1} \\
& =3 F_{2 n+2}-F_{2 n} .
\end{aligned}
$$

Since $F_{0}=1$ and $F_{2}=2$, we have $A_{n}^{\prime}=F_{2 n}$ for all $n \geq 0$, which proves our assertion. The relation between the Fibonacci sequence and the hyperbolic Kac-Moody algebra $g(a)$ was first noticed by Feingold $([\mathrm{F}])$.

Let us find a closed form expression for the sequence $\left\{A_{n}\right\}_{n \geq 0}$. Let $F(x)=$ $\sum_{n=0}^{\infty} A_{n} x^{n}$ be the generating function for the sequence $\left\{A_{n}\right\}_{n \geq 0}$. Then multiplying (2.3) by $x^{n+2}$ yields

$$
A_{n+2} x^{n+2}=a x A_{n+1} x^{n+1}-x^{2} A_{n} x^{n}+x^{n+2}(n \geq 0) .
$$

Summing up over $n \geq 0$ gives

$$
F(x)-A_{0}-A_{1} x=a x\left(F(x)-A_{0}\right)-x^{2} F(x)+\left(x^{2}+x^{3}+\cdots\right),
$$

which yields

$$
\left(1-a x+x^{2}\right) F(x)=x+x^{2}+x^{3}+\cdots=\frac{x}{1-x}
$$

Let

$$
\gamma=\frac{a+\sqrt{a^{2}-4}}{2}
$$

be a zero of $1-a x+x^{2}$. Then

$$
\begin{aligned}
F(x) & =\frac{x}{(1-x)\left(1-a x+x^{2}\right)} \\
& =\frac{-\gamma}{(1-\gamma)^{2}} \frac{1}{1-x}+\frac{\gamma^{2}}{(1+\gamma)(1-\gamma)^{2}} \frac{1}{\gamma-x} \\
& +\frac{\gamma}{(1+\gamma)(1-\gamma)^{2}} \frac{1}{\gamma}-x \\
& =\frac{-\gamma}{(1-\gamma)^{2}} \sum_{n=0}^{\infty} x^{n}+\frac{\gamma}{(1+\gamma)(1-\gamma)^{2}} \sum_{n=0}^{\infty}\left(\frac{x}{\gamma}\right)^{n} \\
& +\frac{\gamma^{2}}{(1+\gamma)(1-\gamma)^{2}} \sum_{n=0}^{\infty}(\gamma x)^{n} \\
& =\sum_{n=0}^{\infty}\left(\frac{-\gamma}{(1-\gamma)^{2}}+\frac{\gamma}{\gamma^{n}(1+\gamma)(1-\gamma)^{2}}+\frac{\gamma^{n+2}}{(1+\gamma)(1-\gamma)^{2}}\right) x^{n}
\end{aligned}
$$




$$
=\sum_{n=0}^{\infty} \frac{1-\gamma^{n}(1+\gamma)+\gamma^{2 n+1}}{\gamma^{n-1}(1+\gamma)(1-\gamma)^{2}} x^{n} .
$$

Therefore, we obtain

$$
A_{n}=\frac{1-\gamma^{n}(1+\gamma)+\gamma^{2 n+1}}{\gamma^{n-1}(1+\gamma)(1-\gamma)^{2}}(n \geq 0) \text {. }
$$

Now we derive the root multiplicity formula for $\mathrm{g}(a)$. For $j \geq 0$, by induction

$$
\begin{aligned}
& r_{0}\left(r_{1} r_{0}\right)^{j} \rho-\rho=-A_{2 j+1} \alpha_{0}-A_{2 j} \alpha_{1}, \\
& r_{1}\left(r_{0} r_{1}\right)^{j} \rho-\rho=-A_{2 j} \alpha_{0}-A_{2 j+1} \alpha_{1}, \\
& \left(r_{0} r_{1}\right)^{j+1} \rho-\rho=-A_{2 j+2} \alpha_{0}-A_{2 j+1} \alpha_{1}, \\
& \left(r_{1} r_{0}\right)^{j+1} \rho-\rho=-A_{2 j+1} \alpha_{0}-A_{2 j+2} \alpha_{1} .
\end{aligned}
$$

It follows from (2.2) and (2.7) that

$$
H=\frac{\sum_{j \geq 0}\left(\mathbf{C}_{-A_{2 j+1} \alpha_{0}-A_{2 j} \alpha_{1}} \oplus \mathbf{C}_{-A_{2 j} \alpha_{0}-A_{2 j+1} \alpha_{1}}\right)}{\sum_{j \geq 0}\left(\mathbf{C}_{-A_{2 j+2} \alpha_{0}-A_{2 j+1} \alpha_{1}} \oplus \mathbf{C}_{-A_{2 j+1} \alpha_{0}-A_{2 j+2} \alpha_{1}}\right)},
$$

and hence for $\tau=-m \alpha_{0}-n \alpha_{1}$, we have

$$
\operatorname{dim} H_{\tau}= \begin{cases}1 & \text { if }(m, n)=\left(A_{2 j+1}, A_{2 j}\right) \text { or }\left(A_{2 j}, A_{2 j+1}\right), \\ -1 & \text { if }(m, n)=\left(A_{2 j+2}, A_{2 j+1}\right) \text { or }\left(A_{2 j+1}, A_{2 j+2}\right), \\ 0 & \text { otherwise. }\end{cases}
$$

For $i, j \geq 0$, define

$$
\tau_{i}= \begin{cases}-A_{2 j+1} \alpha_{0}-A_{2 j} \alpha_{1} & \text { if } i=4 j \\ -A_{2 j} \alpha_{0}-A_{2 j+1} \alpha_{1} & \text { if } i=4 j+1 \\ -A_{2 j+2} \alpha_{0}-A_{2 j+1} \alpha_{1} & \text { if } i=4 j+2 \\ -A_{2 j+1} \alpha_{0}-A_{2 j+2} \alpha_{1} & \text { if } i=4 j+3\end{cases}
$$

Then we have an enumeration of all the weights of $H: P(H)=\left\{\tau_{i} \mid i \geq 0\right\}$, where

$$
\operatorname{dim} H_{\tau_{i}}= \begin{cases}1 & \text { if } i \equiv 0,1(\bmod 4) \\ -1 & \text { if } i \equiv 2,3(\bmod 4)\end{cases}
$$

For $\tau \in Q_{-}$, set

$$
T_{a}(\tau)=\left\{(n)=\left(n_{i}\right)_{i \geq 0} \mid n_{i} \in \mathbf{Z}_{\geq 0}, \sum n_{i} \tau_{i}=\tau\right\},
$$

and define 


$$
B_{a}(\tau)=\sum_{(n) \in T_{a}(\tau)} \frac{\left(\sum n_{i}-1\right) !}{\Pi\left(n_{i} !\right)}(-1)^{\sum_{12 a, 3 \bmod 4:} n_{i}}
$$

Then, by Berman-Moody's formula, we obtain

PROPOSITION 2.1

$$
\operatorname{dimg}(a)_{\alpha}=\sum_{\tau \mid \alpha} \mu\left(\frac{\alpha}{\tau}\right) \frac{\tau}{\alpha} B_{a}(\tau)
$$

Next we examine more closely the formula (2.13) for $B_{a}(\tau)$. Let $\alpha=-n \alpha_{0}$ - $(n+j) \alpha_{1}$ be a root of $\mathrm{g}(a)$ and write $B_{a}(n, n+j)$ for $B_{a}(\alpha)$. By symmetry of the root system, we may suppose without loss of generatity that $j \geq 0$. We noted above that any weight $\tau_{k} \in P(H)$ is of the form

$$
\begin{aligned}
\tau_{k} & =-A_{i} \alpha_{0}-A_{i+1} \alpha_{1} \\
\text { or } \tau_{k} & =-A_{i+1} \alpha_{0}-A_{i} \alpha_{1} .
\end{aligned}
$$

Let

$\mathscr{C}=\left\{\mathbf{c}=\left(c_{0}^{0}, c_{0}^{1}, c_{1}^{0}, c_{1}^{1}, \ldots\right) \mid c_{i}^{j}\right.$ are non-negative integers, $\left.j \in\{0,1\}, j \geq 0\right\}$.

For given $a, n$, and $j$, define

$$
\mathscr{C}(a, n, j)=\left\{\mathbf{c} \in \mathscr{C} \mid \sum_{i=0}^{\infty}\left(c_{\imath}^{0} A_{i+1}+c_{i}^{1} A_{\imath}\right)=n, \sum_{i=0}^{\infty}\left(c_{i}^{0} A_{i}+c_{\imath}^{1} A_{\imath+1}\right)=n+j\right\} .
$$

Then all partitions of $\alpha$ in $T_{a}(\alpha)$ are of the form

$$
\alpha=\sum_{i=0}^{\infty}\left[c_{i}^{0}\left(-A_{i+1} \alpha_{0}-A_{i} \alpha_{1}\right)+c_{i}^{1}\left(-A_{i} \alpha_{0}-A_{i+1} \alpha_{1}\right)\right]
$$

Weights in the partition count with negative multiplicity precisely when $i$ is odd, and so we have

Proposition 2.2

$$
\begin{aligned}
& B_{a}(n, n+j)=\sum_{\mathscr{C}(a, n, j)}(-1)^{\sum_{\text {odd }}\left(c_{i}^{0}+c_{i}^{1}\right)} \frac{\left(\sum_{i \geq 0}\left(c_{i}^{0}+c_{i}^{1}\right)-1\right) !}{\Pi_{i \geq 0} c_{i}^{0} ! c_{i}^{1} !} \\
& =\sum_{\mathscr{C}(a, n, j)}(-1)^{\sum_{\text {odd }}\left(c_{i}^{0}+c_{i}^{1}\right)} \frac{1}{\sum_{i \geq 0}\left(c_{i}^{0}+c_{i}^{1}\right)} \frac{\left(\sum_{i \geq 0}\left(c_{i}^{0}+c_{i}^{1}\right)\right) !}{c_{0}^{0} ! c_{0}^{1} ! c_{1}^{0} ! c_{1}^{1} ! c_{2}^{0} ! \cdots} \\
& =\sum_{\mathscr{C}(a, n, j)}(-1)^{\sum_{\text {odd }}\left(c_{i}^{0}+c_{i}^{1}\right)} \frac{1}{\sum_{i \geq 0}\left(c_{i}^{0}+c_{i}^{1}\right)}\left(\begin{array}{c}
\sum_{i \geq 0}\left(c_{i}^{0}+c_{i}^{1}\right) \\
c_{0}^{0}, c_{0}^{1}, c_{1}^{0}, c_{1}^{1}, \ldots
\end{array}\right) .
\end{aligned}
$$


Example 2.3. Let $a=3$ and consider $\alpha=-4 \alpha_{0}-5 \alpha_{1}$. That is, $n=4$, $j=1$. For $a=3, A_{0}=0, A_{1}=1, A_{2}=4$ and $A_{3}=12>5$. Thus, for any c $\in \mathscr{C}(3,4,1), c_{k}^{0}=c_{k}^{1}=0$ for all $k \geq 2$, and we have the additional two conditions that

$$
\begin{aligned}
& c_{0}^{0}+4 c_{1}^{0}+c_{1}^{1}=4, \\
& c_{0}^{1}+c_{1}^{0}+4 c_{1}^{1}=5 .
\end{aligned}
$$

Hence,

$$
\mathscr{C}(3,4,1)=\{(4,5,0,0, \ldots),(0,4,1,0, \ldots),(3,1,0,1, \ldots)\}
$$

corresponding to the partitions

$$
\begin{aligned}
-4 \alpha_{0}-5 \alpha_{1} & =4\left(-\alpha_{0}\right)+5\left(-\alpha_{1}\right) \\
& =4\left(-\alpha_{1}\right)+\left(-4 \alpha_{0}-\alpha_{1}\right) \\
& =3\left(-\alpha_{0}\right)+\left(-\alpha_{1}\right)+\left(-\alpha_{0}-4 \alpha_{1}\right) .
\end{aligned}
$$

Thus, suppressing trailing zeroes in the multinomials, we have

$$
\begin{aligned}
B_{3}(4,5) & =\frac{1}{9}\left(\begin{array}{c}
9 \\
4,5
\end{array}\right)+(-1)^{1} \frac{1}{5}\left(\begin{array}{c}
5 \\
0,4,1
\end{array}\right)+(-1)^{1} \frac{1}{5}\left(\begin{array}{c}
5 \\
3,1,0,1
\end{array}\right) \\
& =14-1-4=9 .
\end{aligned}
$$

Since no other root $\tau$ divides $\alpha=-4 \alpha_{0}-5 \alpha_{1}$, we have immediately from (2.14) that $\operatorname{dimg}_{\alpha}=9$.

EXAMPLE 2.4. This example gives a foretaste of the stability theory in the following section. Let $\alpha=-n \alpha_{0}-(n+j) \alpha_{1}$. Suppose $a \geq n+j$. Then $A_{2}=a$ $+1>n+j$. Thus, any partition can involve only $A_{0}$ and $A_{1}$. Hence,

$$
\mathscr{C}(a, n, j)=\{(n, n+j, 0,0, \ldots)\},
$$

and

$$
B_{a}(n, n+j)=\frac{1}{2 n+j}\left(\begin{array}{c}
2 n+j \\
n
\end{array}\right)
$$

For $j \neq 0$, if $\operatorname{gcd}(n, n+j)=1$, then $\alpha=-n \alpha_{0}-(n+j) \alpha_{1}$ has no divisors other than itself and

$$
\operatorname{dimg}_{\alpha}=\frac{1}{2 n+j}\left(\begin{array}{c}
2 n+j \\
n
\end{array}\right)
$$


For example, if $j=1$, and $a \geq n+1$, than

$$
\operatorname{dimg}_{\alpha}=\frac{1}{2 n+1}\left(\begin{array}{c}
2 n+1 \\
n
\end{array}\right) .
$$

Thus the multiplicity of, say, $\alpha=-5 \alpha_{0}-6 \alpha_{1}$ in the Kac-Moody algebra $g(a)$ $(a \geq 6)$ is $\operatorname{dimg}(a)_{\alpha}=\frac{1}{11}\left(\begin{array}{c}11 \\ 5\end{array}\right)=42$ (see Table 5 in Section 6). In the next section, we will see that this is actually the same as the multiplicity of $5 \alpha_{0}+6 \alpha_{1}$ in the free Lie algebra of rank 2.

If $j=0$ and $n$ is an odd prime $p$, (2.14) reduces to

$$
\operatorname{dimg}_{\alpha}=\frac{1}{2 p}\left(\begin{array}{c}
2 p \\
p
\end{array}\right)-\frac{1}{p}
$$

Since $\operatorname{dimg}_{\alpha}$ is an integer, this shows that $\left(\begin{array}{c}2 p \\ p\end{array}\right)=2(\bmod 2 p)$.

EXAmple 2.5. Suppose $j=0, n \geq 3$ and $a=n-1$. Then $A_{0}=0, A_{1}=1$, $A_{2}=a+1=n$ and $A_{k}>n$ for $k \geq 3$. Thus, any partition of $\alpha=-n \alpha_{0}-n \alpha_{1}$ can involve only $A_{0}, A_{1}$, and $A_{2}$. Hence, if $\mathbf{c} \in \mathscr{C}(n-1, n, 0), c_{k}^{0}=c_{k}^{1}=0$ for $k \geq 2$. The corresponding partitions of $\alpha$ are:

$$
\begin{aligned}
-n \alpha_{0}-n \alpha_{1} & =n\left(-\alpha_{0}\right)+n\left(-\alpha_{1}\right) \\
& =(n-1)\left(-\alpha_{0}\right)+\left(-\alpha_{0}-n \alpha_{1}\right) \\
& =(n-1)\left(-\alpha_{1}\right)+\left(-n \alpha_{0}-\alpha_{1}\right) .
\end{aligned}
$$

Thus,

$$
\mathscr{C}(n-1, n, 0)=\{(n, n, 0,0, \ldots),(n-1,0,0,1, \ldots),(0, n-1,1,0, \ldots)\},
$$

and

$$
\begin{aligned}
B_{n-1}(n, n) & =\frac{1}{2 n}\left(\begin{array}{c}
2 n \\
n
\end{array}\right)+(-1)^{1} \frac{1}{n}\left(\begin{array}{c}
n \\
1
\end{array}\right)+(-1)^{1} \frac{1}{n}\left(\begin{array}{c}
n \\
1
\end{array}\right) \\
& =\frac{1}{2 n}\left(\begin{array}{c}
2 n \\
n
\end{array}\right)-2 \\
& =B_{n}(n, n)-2 .
\end{aligned}
$$

For example, if $n=5$, we have $B_{4}(5,5)=\frac{252}{10}-2=23 \frac{1}{5}$. Hence, the 
multiplicity of the root $\alpha=-5 \alpha_{0}-5 \alpha_{1}$ in $\mathrm{g}(4)$ is $\operatorname{dimg}_{\alpha}=23 \frac{1}{5}-\frac{1}{5}=23$.

By a similar argument, it is easy to see that, for $n$ sufficiently large, we obtain formulas relating $B_{n-k}(n, n)$ and $B_{n}(n, n)$. The first few are:

$$
\begin{array}{cl}
\text { For } n \geq 3, & B_{n-1}(n, n)=B_{n}(n, n)-2 \\
\text { For } n \geq 4, & B_{n-2}(n, n)=B_{n}(n, n)-2 n+1 \\
\text { For } n \geq 5, & B_{n-3}(n, n)=B_{n}(n, n)-n(n+1)+6 \\
\text { For } n \geq 7, & B_{n-4}(n, n)=B_{n}(n, n)-\frac{n(n+1)(n+2)}{3}+30 \\
\text { For } n \geq 9, & B_{n-5}(n, n)=B_{n}(n, n)-\frac{n(n+1)(n+2)(n+3)}{12} \\
& +140 .
\end{array}
$$

\section{Stability of root multipicities}

In this section, we prove the stability of the root multiplicities of the Kac-Moody algebras $g(a)$, and discuss the relation with the free Lie algebra with 2 generators. We start with the discussion on free Lie algebras. Let $X=\{x \mid i=$ $1,2,3, \ldots\}$ be a totally ordered set (possibly countably infinite) and let $R$ be an (additive) partially ordered abelian semigroup with a countable basis such that each element $\alpha$ of $R$ can be expressed as a sum of elements of $R$ which are less than or equal to $\alpha$ in only finitely many ways. Let $G$ be the free Lie algebra on the set $X$. We make $G$ an $R$-graded Lie algebra as follows. Let $\mathscr{S}=\left\{\mu_{i} \mid i=1,2,3\right.$, ...) be a collection of elements in $R$ such that $\mu_{\imath} \leq \mu_{j}$ for $i<j$. We allow only finitely many repetitions. Define $\operatorname{deg} x_{i}=\mu_{i}$, and

$$
\operatorname{deg}\left[\left[\cdots\left[x_{i_{1}}, x_{i_{2}}\right], \cdots\right] x_{i_{r}}\right]=\mu_{i_{1}}+\mu_{i_{2}}+\cdots+\mu_{i_{r}} .
$$

Then $G$ becomes an $R$-graded Lie algebra $G=\bigoplus_{\alpha \in R} G_{\alpha}$, where $G_{\alpha}$ is the subspace of $G$ spanned by all the brackets $\left[\left[\cdots\left[x_{i_{1}}, x_{i_{2}}\right], \cdots\right] x_{i_{r}}\right]$ such that $\mu_{i_{1}}+\mu_{i_{2}}$ $+\cdots+\mu_{i_{r}}=\alpha$.

We recall the dimension formula for $G_{\alpha}$ obtained in [Ka1]. Let $P=\left\{\tau_{i} \mid i=\right.$ $1,2,3, \ldots\}$ be the set of distinct elements in $\mathscr{S}$, and let $H$ be the subspace of $G$ spanned by the elements of $X$. Then $H$ has the decomposition $H=\bigoplus_{i=1}^{\infty} H_{\tau_{i}}$. For $\tau$ $\in R$, let

$$
T_{0}(\tau)=\left\{(n)=\left(n_{i}\right)_{i \geq 1} \mid n_{i} \in \mathbf{Z}_{\geq 0}, \sum n_{i} \tau_{i}=\tau\right\}
$$


and define

$$
B_{0}(\tau)=\sum_{(n) \in T(\tau)} \frac{\left(\sum n_{i}-1\right) !}{\Pi n_{i} !} \Pi\left(\operatorname{dim} H_{\tau_{i}}\right)^{n_{i}}
$$

Then by generalizing the proof of the Witt formula given in [Se], we obtain

PROPOSITION 3.1 ([Ka1]).

$$
\operatorname{dim} G_{\alpha}=\sum_{\tau \mid \alpha} \mu\left(\frac{\alpha}{\tau}\right) \frac{\tau}{\alpha} B_{0}(\tau)
$$

Fix the index set $I=\{1,2, \cdots, n\}$ and let $\mathfrak{F}$ be the free Lie algebra generated by the elements $e_{i}(i=1, \cdots, n)$. Since $\operatorname{deg} e_{\imath}=\alpha_{i}$ for $i=1, \cdots, n, \mathfrak{F}$ is a $Q_{+}$-graded Lie algebra $\mathfrak{F}=\bigoplus_{\alpha \in Q_{+}} \mathfrak{F}_{\alpha}$, where $\mathfrak{F}_{\alpha}$ is the subspace of $\mathfrak{F}$ spanned by all the brackets $\left[\left[\cdots\left[e_{i_{1}}, e_{i_{2}}\right], \cdots\right] e_{i_{r}}\right]$ such that $\alpha_{i_{1}}+\alpha_{i_{2}}+\cdots+\alpha_{i_{r}}=\alpha$. For $\tau$ $=\sum_{i=1}^{n} t_{i} \alpha_{\imath} \in Q_{+}$, let $h t(\tau)=\sum_{i=1}^{n} t_{i}$, and define

$$
B_{\infty}(\tau)=\frac{\left(\sum t_{i}-1\right) !}{\Pi t_{i} !}=\frac{1}{\operatorname{ht}(\tau)}\left(\begin{array}{c}
\operatorname{ht}(\tau) \\
t_{1}, \cdots, t_{n}
\end{array}\right) .
$$

Then Proposition 3.1 implies

Corollary 3.2

$$
\operatorname{dim} \mathfrak{F}_{\alpha}=\sum_{\tau \mid \alpha} \mu\left(\frac{\alpha}{\tau}\right) \frac{\tau}{\alpha} B_{\infty}(\tau)
$$

Proof. For $\tau=\sum_{i=1}^{n} t_{i} \alpha_{i} \in Q_{+}$, the only partition of $\tau$ is

$$
\tau=t_{1}\left(\alpha_{1}\right)+\cdots+t_{n}\left(\alpha_{n}\right) .
$$

Now the result follows immediately from Proposition 3.1.

EXAmple 3.3 Let $\mathfrak{F}$ be the free Lie algebra generated by the elements $e_{0}$ and $e_{1}$. For $\tau=m \alpha_{0}+m \alpha_{1}$, we write $\tau=(m, n)$. By (3.4), we have

$$
B_{\infty}(m, n)=\frac{1}{m+n}\left(\begin{array}{c}
m+n \\
m
\end{array}\right) \text {. }
$$

Therefore, if $\alpha=5 \alpha_{0}+6 \alpha_{1}$, then

$$
\operatorname{dim} \mathfrak{F}_{\alpha}=B_{\infty}(5,6)=\frac{1}{11}\left(\begin{array}{c}
11 \\
5
\end{array}\right)=42 .
$$


If $\alpha=4 \alpha_{0}+6 \alpha_{0}$, then

$$
\operatorname{dim} \mathfrak{F}_{\alpha}=B_{\infty}(4,6)-\frac{1}{2} B_{\infty}(2,3)=\frac{1}{10}\left(\begin{array}{c}
10 \\
4
\end{array}\right)-\frac{1}{2} \frac{1}{5}\left(\begin{array}{l}
5 \\
2
\end{array}\right)=20 .
$$

We now discuss the stability of root multiplicities for general symmetrizable Kac-Moody algebras. Let $A=\left(a_{i j}\right)_{i, j \in I}$ and $A^{\prime}=\left(a_{i j}^{\prime}\right)_{i, j \in I}$ be generalized Cartan matrices of the same size. We define $A \leq A^{\prime}$ if $\left|a_{i j}\right| \leq\left|a_{i j}^{\prime}\right|$ for all $i \neq j$. We write $A>0$ if $\left|a_{i j}\right|>0$ for all $i \neq j$.

Proposition 3.4. Suppose $A \leq A^{\prime}$, and let $\alpha \in Q$ be a root of the Kac-Moody algebra $\mathrm{g}=\mathrm{g}(A)$. Then $\alpha$ is also a root of the Kac-Moody algebra $\mathrm{g}^{\prime}=\mathrm{g}\left(A^{\prime}\right)$, and we have $\operatorname{dim} g_{\alpha} \leq \operatorname{dim} g_{\alpha}^{\prime}$.

Proof. We may assume that $\alpha \in Q_{+}$. We denote by $e_{i}^{\prime}, f_{i}^{\prime}(i \in I)$ the Chevalley generators of $g^{\prime}$. Let $g_{+}$(resp. $\left.g_{+}^{\prime}\right)$ be the subalgebra of $g$ (resp. $e^{\prime}$ ) generated by the elements $e_{i}$ (resp. $e_{i}^{\prime}$ ) for $i \in I$. Since $\left|a_{i j}\right| \leq\left|a_{i j}^{\prime}\right|$ for all $i \neq j\left(A \leq A^{\prime}\right)$, by Gabber-Kac Theorem ([GK]), there is a surjective Lie algebra homomorphism $\phi_{A^{\prime}, A}: \mathfrak{g}_{+}^{\prime} \rightarrow g_{+}$defined by $e_{i}^{\prime} \mapsto e_{i}$. Therefore $\operatorname{dimg}_{\alpha} \leq \operatorname{dimg}_{\alpha}^{\prime}$ for all $\alpha \in Q_{+}$.

CoRollary 3.5. If $a \leq a^{\prime}$, them $\operatorname{dimg}(a)_{\alpha} \leq \operatorname{dimg}\left(a^{\prime}\right)_{\alpha}$.

THEOREM 3.6. Let $\alpha=\sum_{i \in I} k_{i} \alpha_{i} \in Q_{+}$, and let $\mathrm{g}=\mathrm{g}(A)$ be the Kac-Moody algebra associated with a generalized Cartan matrix $A=\left(a_{i j}\right)_{i, j \in I}$. Then $\operatorname{dimg}_{\alpha}$ is the same for all $A \gg 0$. Moreover, if $A \gg 0$, we have

$$
\operatorname{dimg}_{\alpha}=\sum_{\tau \mid \alpha} \mu\left(\frac{\alpha}{\tau}\right) \frac{\tau}{\alpha} B_{\infty}(\tau)
$$

where $B_{\infty}(\tau)$ is defined by $(3.4)$.

Proof. We will consider $\operatorname{dimg}_{-\alpha}$. We take $S=\phi$ and apply Berman-Moody's formula. By Kostant's formula, we have

$$
\begin{aligned}
& H_{1}\left(\mathrm{~g}_{-}\right)=\bigoplus_{i \in I} \mathbf{C}_{r_{i} \rho-\rho}=\bigoplus \mathbf{C}_{-\alpha_{i}}, \\
& H_{2}\left(\mathrm{~g}_{-}\right)=\bigoplus_{\substack{i, j \in I \\
i \neq j}} \mathbf{C}_{r_{i} r_{j} \rho-\rho}=\bigoplus_{\substack{i, j \in I \\
i \neq j}} \mathbf{C}_{-\alpha_{j}-\left(1-a_{i j}\right) \alpha_{i}}
\end{aligned}
$$

Therefore 


$$
P(H)=\left\{-\alpha_{i} \mid i \in I\right\} \cup\left\{-\alpha_{j}-\left(1-a_{i j}\right) \alpha_{i} \mid i, j \in I, i \neq j\right\} \cup \cdots \cdots .
$$

Since $A \gg 0$, for any $\tau=\sum_{i \in I} t_{i} \alpha_{i}$ that divides $\alpha$, there is only one partition of - $\tau$ into a sum of the elements in $P(H)$ :

$$
-\tau=t_{1}\left(-\alpha_{1}\right)+\cdots+t_{n}\left(-\alpha_{n}\right)
$$

and $\operatorname{dim} H_{-\alpha_{i}}=1$ for all $i \in I$. Hence we obtain

$$
\operatorname{dimg}_{\alpha}=\sum_{\tau \mid \alpha} \mu\left(\frac{\alpha}{\tau}\right) \frac{\tau}{\alpha} \frac{\left(\sum t_{i}-1\right) !}{\Pi t_{i} !}=\sum_{\tau \mid \alpha} \mu\left(\frac{\alpha}{\tau}\right) \frac{\tau}{\alpha} B_{\infty}(\tau) .
$$

Remark. We can summarize the above discussion as follows. By Proposition 3.4 , we have a projective system $\left\{g_{+}(A), \phi_{A^{\prime}, A}\right\}$. It follows from Corollary 3.2 and Theorem 3.6 that the projective limit of the above system is the free Lie algebra $\mathfrak{F}$ generated by the elements $e_{i}(i=1, \ldots, n)$. We may consider $\mathfrak{F}$ as the subalgebra of the Kac-Moody algebra $g(A)$ generated by the elements $e_{i}(i=1, \ldots, n)$, where the Cartan matrix $A=\left(a_{i j}\right)$ is given by $a_{i j}=-\infty$ for all $i \neq j$. That is, in the rank 2 case, we may consider $\mathfrak{F}$ as $\mathfrak{g}_{+}(\infty)$.

In the following example, we illustrate the stability of root multiplicities for the root $\alpha=5 \alpha_{0}+6 \alpha_{1}$. Compare with Example 2.4 and Example 3.3.

\section{EXAMPLE 3.7.}

(a) Let $a=3$. Then by (2.3) we have

$$
A_{0}(3)=0, A_{1}(3)=1, A_{2}(3)=4, A_{3}(3)=12, \cdots \cdots,
$$

and hence

$$
\mathscr{C}(3,5,6)=\{(5,6,0,0, \cdots),(1,5,1,0, \cdots),(4,2,0,1, \cdots),(0,1,1,1, \cdots)\},
$$

corresponding to the partitions

$$
\begin{aligned}
-5 \alpha_{0}-6 \alpha_{1} & =5\left(-\alpha_{0}\right)+6\left(-\alpha_{1}\right) \\
& =\left(-\alpha_{0}\right)+5\left(-\alpha_{1}\right)+\left(-4 \alpha_{0}-\alpha_{1}\right) \\
& =4\left(-\alpha_{0}\right)+2\left(-\alpha_{1}\right)+\left(-\alpha_{0}-4 \alpha_{1}\right) \\
& =\left(-\alpha_{0}\right)+\left(-4 \alpha_{0}-\alpha_{1}\right)+\left(-\alpha_{0}-4 \alpha_{1}\right) .
\end{aligned}
$$

It follows that

$$
B_{3}(5,6)=\frac{1}{11}\left(\begin{array}{c}
11 \\
5,6
\end{array}\right)+(-1)^{1} \frac{1}{7}\left(\begin{array}{c}
7 \\
1,5,1
\end{array}\right)+(-1)^{1} \frac{1}{7}\left(\begin{array}{c}
7 \\
4,2,0,1
\end{array}\right)
$$




$$
+(-1)^{2} \frac{1}{3}\left(\begin{array}{c}
3 \\
1,1,1
\end{array}\right)=23
$$

Therefore $\operatorname{dimg}(3)_{\alpha}=B_{3}(5,6)=23$.

(b) If $a=4$, then we have

$$
A_{0}(4)=0, A_{1}(4)=1, A_{2}(4)=5, A_{3}(4)=20, \cdots \cdots,
$$

and

$$
\mathscr{C}(4,5,6)=\{(5,6,0,0, \cdots),(0,5,1,0, \cdots),(4,1,0,1, \cdots)\}
$$

Therefore

$$
\begin{aligned}
\operatorname{dimg}(4)_{\alpha} & =B_{4}(5,6)=\frac{1}{11}\left(\begin{array}{c}
11 \\
5,6
\end{array}\right)+(-1)^{1} \frac{1}{6}\left(\begin{array}{c}
6 \\
5,1
\end{array}\right) \\
& +(-1)^{1} \frac{1}{6}\left(\begin{array}{c}
6 \\
4,1,0,1
\end{array}\right)=36
\end{aligned}
$$

(c) If $a=5$, then we have

$$
A_{0}(5)=0, A_{1}(5)=1, A_{2}(5)=6, A_{3}(5)=30, \cdots \cdots,
$$

and

$$
\mathscr{C}(5,5,6)=\{(5,6,0,0, \cdots),(4,0,0,1, \cdots)\}
$$

Therefore

$$
\operatorname{dimg}(5)_{\alpha}=B_{5}(5,6)=\frac{1}{11}\left(\begin{array}{c}
11 \\
5,6
\end{array}\right)+(-1)^{1} \frac{1}{5}\left(\begin{array}{c}
5 \\
4,0,0,1
\end{array}\right)=41
$$

(d) If $a=6$, then we have

$$
A_{0}(6)=0, A_{1}(6)=1, A_{2}(6)=7, A_{3}(6)=42, \cdots \cdots,
$$

and

$$
\mathscr{C}(6,5,6)=\{(5,6,0,0, \cdots)\}
$$

Therefore

$$
\operatorname{dimg}(6)_{\alpha}=B_{4}(5,6)=\frac{1}{11}\left(\begin{array}{c}
11 \\
5,6
\end{array}\right)=42 .
$$

Clearly, for $\alpha \geq 6$, we have

$$
\mathscr{C}(a, 5,6)=\{(5,6,0,0, \cdots)\},
$$


and hence $\operatorname{dimg}(a)_{\alpha}=B_{a}(5,6)=42$ (see Table 7 in Section 6$)$.

\section{The root system of the algebra $\mathfrak{g}(a)$}

In this section, we study the root system of the algebra $\mathrm{g}(a)$, and give an explicit description of the real roots and imaginary roots. As a by-product, we obtain a simple algorithm to find the integral points on certain hyperbolas.

A root $\alpha$ of a Kac-Moody algebra $g(A)$ is called real if $\alpha=w\left(\alpha_{i}\right)$ for some $w \in W$ and $i \in I$. A root that is not real is called imaginary. We denote by $\Delta^{\text {re }}$, $\Delta_{+}^{\mathrm{re}}, \Delta^{\mathrm{im}}$, and $\Delta_{+}^{\mathrm{im}}$ the set of real roots, positive real roots, imaginary roots, and positive imaginary roots, respectively. We recall some of the fundamental properties of the imaginary roots.

Proposition 4.1 ([K]).

(a) The set $\Delta_{+}^{\mathrm{im}}$ is $W$-invariant.

(b) For $\alpha \in \Delta_{+}^{\mathrm{im}}$, there exists a unique $\beta \in \Delta_{+}^{\mathrm{im}}$ such that $\beta=w(\alpha)$ for some $w \in W$ and $\beta\left(h_{i}\right) \leq 0$ for all $i \in I$.

For a Kac-Moody algebra $g(A)$ with a symmetrizable generalized Cartan matrix $A=\left(a_{i j}\right)_{i, j \in I}$, there is a nondegenerate symmetric bilinear form $(\mid)$ on $\mathfrak{h}^{*}$ such that $a_{i j}=\frac{2\left(\alpha_{i} \mid \alpha_{j}\right)}{\left(\alpha_{i} \mid \alpha_{i}\right)}$ for all $i, j \in I$. If $A$ is symmetric, we take $\left(\alpha_{i} \mid \alpha_{i}\right)=2$ for all $i \in I$ so that $a_{i j}=\left(\alpha_{i} \mid \alpha_{j}\right)$ for all $i, j \in I$.

A generalized Cartan matrix $A$ is said to be of finite type if all of its principal minors are positive, of affine type if all of its proper principal submatrices are of finite type and $\operatorname{det} A=0$, and of indefinite type otherwise. $A$ is of hyperbolic type if it is of indefinite type and all of its proper principal submatrices are either of finite type or of affine type. The corresponding Kac-Moody algebra $g(A)$ is called a finite, affine, indefinite, or hyperbolic Kac-Moody algebra, respectively. The following proposition gives a nice description of the root system of hyperbolic Kac-Moody algebras.

Proposition $4.2([\mathrm{~K}],[\mathrm{M}])$. Let $\mathrm{g}(A)$ be a hyperbolic Kac-Moody algebra with a symmetric generalized Cartan matrix.Then we have:

(a) $\Delta^{\text {re }}=\{\alpha \in Q \mid(\alpha \mid \alpha)=2\}$

(b) $\Delta^{\mathrm{im}}=\{\alpha \in Q \backslash\{0\} \mid(\alpha \mid \alpha) \leq 0\}$. 
We now focus on the structure of the algebra $\mathfrak{g}(a)$. We assume $a \geq 3$. Then the algebra $\mathfrak{g}(a)$ is of hyperbolic type. We identify an element $\alpha=x \alpha_{0}+y \alpha_{1}$ $\in Q$ with an integral point $(x, y) \in \mathbf{Z} \times \mathbf{Z}$. We call $\alpha=(x, y) \in \mathbf{Z} \times \mathbf{Z}$ a positive integral point if $x, y \in \mathbf{Z}_{\geq 0}$. Define a symmetric bilinear form $(\mid)$ on $\mathfrak{h}^{*}$ by

$$
\begin{aligned}
& \left(\alpha_{0} \mid \alpha_{0}\right)=\left(\alpha_{1} \mid \alpha_{1}\right)=2, \\
& \left(\alpha_{0} \mid \alpha_{1}\right)=-\alpha .
\end{aligned}
$$

Then for $\alpha=(x, y) \in \mathbf{Z} \times \mathbf{Z}$, we have $(\alpha \mid \alpha)=2\left(x^{2}-a x y+y^{2}\right)$. Therefore, as an immediate consequence of Proposition 4.2, we obtain:

COROLlary 4.3. For $a \geq 3$, the root system of the algebra $\mathrm{g}(a)$ is given by

(a) $\Delta^{\mathrm{re}}=\left\{(x, y) \in \mathbf{Z} \times \mathbf{Z} \mid x^{2}-a x y+y^{2}=1\right\}$,

(b) $\Delta^{\mathrm{im}}=\left\{(x, y) \in \mathbf{Z} \times \mathbf{Z} \mid x^{2}-a x y+y^{2} \leq 0\right\}$.

In particular, there is a one-to-one correspondence between the set of real roots of $\mathrm{g}(a)$ and the set of all integral points on the hyperbola $\mathscr{H}^{1}: x^{2}-a x y+$ $y^{2}=1$. Since there are no integral points on the union of lines $x^{2}-a x y+y^{2}=$ 0 , the imaginary roots of $g(a)$ correspond to the integral points on the hyperbolas $\mathscr{H}_{k}: x^{2}-a x y+y^{2}=-k$ for $k \geq 1$. In other words, for each $k \geq 1$, there is a one-to-one correspondence between the set of all imaginary roots $\alpha$ with lenghth $(\alpha \mid \alpha)=-2 k$ and the set of all integral points on the hyperbola $\mathscr{H}_{k}$.

To describe the root system of the algebra $\mathfrak{g}(a)$, we introduce a sequence $\left\{B_{n}\right\}_{n \geq 0}$ defined by

$$
\begin{gathered}
B_{0}=0, \quad B_{1}=1 \\
B_{n+2}=a B_{n+1}-B_{n} \text { for } n \geq 0 .
\end{gathered}
$$

If $a=3$, then we have $B_{n}=F_{2 n-1}$ for all $n \geq 1$, where $\left\{F_{n}\right\}$ is the Fibonacci sequence defined by (2.4). By a similar method used in Section 2, we obtain a closed form expression for the sequence $\left\{B_{n}\right\}_{n \geq 0}$ :

$$
B_{n}=\frac{1-\gamma^{2 n}}{\gamma^{n-1}\left(1-\gamma^{2}\right)}(n \geq 0) \text {, }
$$

where $\gamma=\frac{a+\sqrt{a^{2}-4}}{2}$ is a zero of $1-a x+x^{2}$. We can directly check that the points $\left(B_{n}, B_{n+1}\right)$ lie on the hyperbola $\mathscr{H}^{1}: x^{2}-a x y+y^{2}=1$ for all $n \geq 0$. Since the hyperbola $\mathscr{H}^{1}$ is asymptotic to the line $y=\gamma x$, it follows that 


$$
B_{n+1}=\left\lceil r B_{n}\right\rceil \text { for all } n \geq 0,
$$

where $\lceil x\rceil$ denotes the smallest integer $\geq x$.

For $j \geq 0$, induction, we have

$$
\begin{aligned}
& r_{0}\left(r_{1} r_{0}\right)^{j}\left(\alpha_{0}\right)=-B_{2 j+1} \alpha_{0}-B_{2 j} \alpha_{1}, \\
& r_{1}\left(r_{0} r_{1}\right)^{j}\left(\alpha_{0}\right)=B_{2 j+1} \alpha_{0}+B_{2 j+2} \alpha_{1}, \\
& \left(r_{0} r_{1}\right)^{j+1}\left(\alpha_{0}\right)=B_{2 j+3} \alpha_{0}+B_{2 j+2} \alpha_{1}, \\
& \left(r_{1} r_{0}\right)^{j+1}\left(\alpha_{0}\right)=-B_{2 j+1} \alpha_{0}-B_{2 j+2} \alpha_{1},
\end{aligned}
$$

and

$$
\begin{aligned}
& r_{0}\left(r_{1} r_{0}\right)^{j}\left(\alpha_{1}\right)=B_{2 j+2} \alpha_{0}+B_{2 j+1} \alpha_{1}, \\
& r_{1}\left(r_{0} r_{1}\right)^{j}\left(\alpha_{1}\right)=-B_{2 j} \alpha_{0}-B_{2 j+1} \alpha_{1}, \\
& \left(r_{0} r_{1}\right)^{j+1}\left(\alpha_{1}\right)=-B_{2 j+2} \alpha_{0}-B_{2 j+1} \alpha_{1}, \\
& \left(r_{1} r_{0}\right)^{j+1}\left(\alpha_{1}\right)=B_{2 j+2} \alpha_{0}+B_{2 j+3} \alpha_{1} .
\end{aligned}
$$

Hence we obtain:

Proposition 4.4 (cf. [BKM], [F]). For $a \geq 3$, the set of all positive real roots of the algebra $\mathrm{g}(a)$ is

$$
\Delta_{+}^{\mathrm{re}}=\left\{\left(B_{n}, B_{n+1}\right),\left(B_{n+1}, B_{n}\right) \mid n \geq 0\right\} .
$$

The correspondence established in [LM] between the root the systems of rank 2 Kac-Moody algebras and quasi-regular cusps reveals more of the geometric nature of the root system. In particular, one should see [LM, Theorem 4.1], which characterizes the real roots as a support polygon.

We now consider the set of imaginary roots of $g(a)$. For a positive integer $k$, let $\Delta_{+, k}^{\mathrm{im}}$ be the set of all positive imaginary roots $\alpha$ of $\mathrm{g}(a)$ with length $(\alpha \mid \alpha)=$ $-2 k$. That is, $\Delta_{+, k}^{\mathrm{im}}$ is the set of all positive integral points on the hyperbola $\mathscr{H}_{k}$ : $x^{2}-a x y+y^{2}=-k$. For any $\alpha \in \Delta_{+, k}^{\text {im }}$, by Proposition 4.1 , there is a unique $\beta=(m, n) \in \Delta_{+}^{\text {im }}$ such that $\beta=w(\alpha)$ for some we $W \in W$ and

$$
\begin{aligned}
& \beta\left(h_{0}\right)=2 m-a n \leq 0, \\
& \beta\left(h_{1}\right)=-a m+2 n \leq 0 .
\end{aligned}
$$

Since the bilinear form $(\mid)$ is $W$-invariant, we also have $\beta \in \Delta_{+, k}^{\mathrm{im}}$. Let $\Omega_{k}$ be the 
set of all positive integral points $\beta=(m, n)$ on the hyperbola $\mathscr{H}_{k}$ that lie between the lines $y=x$ and $y=\frac{a x}{2}$, and let $\overline{\Omega_{k}}$ be the mirror image of the set $\Omega_{k}$ with respect to the line $y=x$. Then, by Proposition 4.1 and the symmetry of the root system, we have

$$
\Delta_{+, k}^{\mathrm{im}}=\left(W \cdot \Omega_{k}\right) \cup\left(W \cdot \overline{\Omega_{k}}\right) .
$$

The hyperbola $\mathscr{H}_{k}$ and the line $y=\frac{a x}{2}$ meet at the point $P_{k}=\left(\frac{2 \sqrt{k}}{\sqrt{a^{2}-4}}\right.$, $\left.\frac{a \sqrt{k}}{\sqrt{a^{2}-4}}\right)$. Also, the tangent line to the hyperbola $\mathscr{H}_{k}$ at the point $P_{k}$ is the line $x=\frac{2 \sqrt{k}}{\sqrt{a^{2}-4}}$. On the other hand, the line $y=x$ meets the hyperbola $\mathscr{H}_{k}$ at the point $\left(\sqrt{\frac{k}{a-2}}, \sqrt{\frac{k}{a-2}}\right)$. Hence we obtain

$$
\begin{gathered}
\Omega_{k}=\left\{(m, n) \mathbf{Z}_{\geq 0} \times \mathbf{Z}_{\geq 0} \mid \frac{2 \sqrt{k}}{\sqrt{a^{2}-4}} \leq m \leq \sqrt{\frac{k}{a-2}},\right. \\
\left.n=\frac{a m-\sqrt{\left(a^{2}-4\right) m^{2}-4 k}}{2}\right\} .
\end{gathered}
$$

THEOREM 4.5. For $a \geq 3$, the set of all positive imaginary roots of the algebra $\mathrm{g}(a)$ with length $-2 k$ is

$$
\begin{aligned}
& \Delta_{+, k}^{\mathrm{im}}=\left\{\left(m B_{j+1}-n B_{j}, m B_{j+2}-n B_{j+1}\right),\right. \\
& \left(m B_{j+2}-n B_{j+1}, m B_{j+1}-n B_{j}\right),\left(n B_{j+1}-m B_{j}, n B_{j+2}-m B_{j+1}\right), \\
& \left.\left(n B_{j+2}-m B_{j+1}, n B_{j+1}-m B_{j}\right) \mid(m, n) \in \Omega_{k}\right\} .
\end{aligned}
$$

Remark. Theorem 4.5 provides us with a simple algorithm to find all the integral points on the hyperbola $\mathscr{H}_{k}: x^{2}-a x y+y^{2}=-k(k \geq 1)$, as is illustrated in the following example.

EXAmple 4.6. Let $a=3$. Thus we have $B_{n}=F_{2 n-1}(n \geq 1)$, where $\left\{F_{n}\right\}$ is the Fibonacci sequence defined by (2.4).

(a) If $k=1$, then we have 


$$
\frac{2 \sqrt{k}}{\sqrt{a^{2}-4}}=\frac{2}{\sqrt{5}}=0.89 \cdots, \text { and } \sqrt{\frac{k}{a-2}}=1 \text {. }
$$

Hence $m=1$ is the only integer in the interval $\frac{2}{\sqrt{5}} \leq m \leq 1$. For $m=1$, we have $n=1$. Therefore $\Omega_{1}=\{(1,1)\}$, and hence the set of all integral points on the hyperbola $x^{2}-3 x y+y^{2}=-1$ is

$$
\begin{aligned}
& \left\{\left(B_{j+1}-B_{j}, B_{j+2}-B_{j+1}\right),\left(B_{j+2}-B_{j+1}, B_{j+1}-B_{j}\right),\right. \\
& \left.\left(B_{j}-B_{j+1}, B_{j+1}-B_{j+2}\right),\left(B_{j+1}-B_{j+2}, B_{j}-B_{j+1}\right) \mid j \geq 0\right\} .
\end{aligned}
$$

(b) If $k=2$, then

$$
\frac{2 \sqrt{k}}{\sqrt{a^{2}-4}}=\frac{2 \sqrt{2}}{\sqrt{5}}=1.26 \cdots, \text { and } \sqrt{\frac{k}{a-2}}=\sqrt{2}=1.14 \cdots .
$$

Thus $\Omega_{2}=\phi$, and hence there is no integral point on the hyperbola $x^{2}-3 x y+$ $y^{2}=-2$.

(c) If $k=100$, then

$$
\frac{2 \sqrt{k}}{\sqrt{a^{2}-4}}=\frac{20}{\sqrt{5}}=8.94 \cdots, \text { and } \sqrt{\frac{k}{a-2}}=10 .
$$

Hence $m=9$ and $m=10$ are all the integers in the interval $\frac{20}{\sqrt{5}} \leq m \leq 10$. If $m=9$, then $n=\frac{27-\sqrt{5}}{2} \notin \mathbf{Z}_{+}$, and if $m=10$, then $n=10$. Therefore $\Omega_{100}$ $=\{(10,10)\}$, and hence the set of all integral points on the hyperbola $x^{2}-3 x y$ $+y^{2}=-100$ is

$$
\begin{aligned}
& \left\{\left(10 B_{j+1}-10 B_{j}, 10 B_{j+2}-10 B_{j+1}\right),\left(10 B_{j+2}-10 B_{j+1}, 10 B_{j+1}-10 B_{j}\right),\right. \\
& \left.\left(10 B_{j}-10 B_{j+1}, 10 B_{j+1}-10 B_{j+2}\right),\left(10 B_{j+1}-10 B_{j+2}, 10 B_{j}-10 B_{j+1}\right) \mid j \geq 0\right\} .
\end{aligned}
$$

(d) If $k=121$, then

$$
\frac{2 \sqrt{k}}{\sqrt{a^{2}-4}}=\frac{22}{\sqrt{5}}=9.83 \cdots, \text { and } \sqrt{\frac{k}{a-2}}=11 .
$$

Hence $m=10$ and $m=11$ are all the integers in the interval $\frac{22}{\sqrt{5}} \leq m \leq 11$. If $m=10$, then $n=13$, and if $m=11$, then $n=11$. Therefore $\Omega_{121}=\{(10,13)$, $(11,11)\}$, and hence the set of all integral points on the hyperbola $x^{2}-3 x y-y^{2}$ $=-121$ is 


$$
\begin{aligned}
& \left\{\left(10 B_{j+1}-13 B_{j}, 10 B_{j+2}-13 B_{j+1}\right),\left(10 B_{j+2}-13 B_{j+1}, 10 B_{j+1}-13 B_{j}\right),\right. \\
& \left(13 B_{j}-10 B_{j+1}, 13 B_{j+1}-10 B_{j+2}\right),\left(13 B_{j+1}-10 B_{j+2}, 13 B_{j}-10 B_{j+1}\right), \\
& \left(11 B_{j+1}-11 B_{j}, 11 B_{j+2}-11 B_{j+1}\right),\left(11 B_{j+2}-11 B_{j+1}, 11 B_{j+1}-11 B_{j}\right), \\
& \left(11 B_{j}-11 B_{j+1}, 11 B_{j+1}-11 B_{j+2}\right),\left(11 B_{j+1}-11 B_{j+2}, 11 B_{j}-11 B_{j+1}\right) \mid \\
& j \geq 0\}
\end{aligned}
$$

\section{Root multiplicity relationships}

In this section, we explain some of the relationships among the root multiplicities of the hyperbolic Kac-Moody algebras $g(a)$. In Section 3, we considered how the multiplicity of a given root varied with the algebra; here, we will restrict to a single algebra at a time and examine relationships between the multiplicities of different roots of that algebra.

\section{\$5.1. Column symmetry}

We look first at the symmetry apparent in the columns of Tables 1-4. That is, fix an algebra $\mathrm{g}=\mathrm{g}(a)$, fix $n$ and consider the multiplicities of the roots $-n \alpha_{0}-(n+j) \alpha_{1}$ as $j$ varies in $\mathbf{Z}$. We begin with a result of Kac [K, Proposition 3.6].

Proposition $5.1([\mathrm{~K}])$. Let $V$ be a finite-dimensional module over $\mathfrak{B} l_{2}$, and let $\lambda$ be a weight of $V$. Denote by $M$ the set of all $t \in \mathbf{Z}$ such that $\lambda+t \alpha$ is a weight of $V$, where $\alpha$ is the simple root of $\& l_{2}$. Let $m_{t}=\operatorname{mult}_{V}(\lambda+t \alpha)$. Then

(a) $M$ is the closed interval of integers $[-p, q]$, where $p, q \in \mathbf{Z}_{+}$and $p-q$ $=\lambda(h)$.

(b) The function $t \mapsto m_{t}$ is increasing on the interval $\left[-p, \frac{1}{2} \lambda(h)\right]$ and is symmetric with respect to $t=\frac{1}{2} \lambda(h)$.

With $a$ and $n$ fixed, let $V=\bigoplus_{j \in \mathbf{Z}} \mathfrak{g}_{-\left(n \alpha_{0}+(n+j) \alpha_{1}\right)}$. Then $V$ is a finitedimensional module over the subalgebra $g_{0}$ of $g(a)$ generated by $e_{1}, f_{1}, h_{1}$. That is, $g_{0} \cong \mathfrak{g} l_{2}$. Let $\lambda=-n \alpha_{0}-n \alpha_{1}$. Then $\lambda\left(h_{1}\right)=n(a-2)$ and we can use part (b) of Proposition 5.1 to find the location of the maximal values of mult $\left(\lambda+t \alpha_{1}\right)$. The result divides into three cases according as $a$ and $n$ are even or odd.

(1) If $a$ is even, then $-\frac{1}{2} \lambda\left(h_{1}\right)=-\frac{1}{2} n(a-2) \in \mathbf{Z}$ and hence the maximal 
root multiplicity occurs for the root $\lambda-\frac{1}{2} n(a-2) \alpha_{1}=-n \alpha_{0}-$ $\frac{1}{2} a n \alpha_{1}$

(2) If $a$ is odd and $n=2 m$ is even, then $-\frac{1}{2} \lambda\left(h_{1}\right)=-m(a-2) \in \mathbf{Z}$ and the maximum root multiplicity occurs for the root $\lambda-m(a-2) \alpha_{1}=$ $-2 m \alpha_{0}-a m \alpha_{1}$.

(3) If $a$ is odd and $n=2 m+1$ is odd, then $-\frac{1}{2} \lambda\left(h_{1}\right)=-\frac{1}{2}(2 m+1)$. $(a-2) \notin \mathbf{Z}$, and the closest integers to $-\frac{1}{2} \lambda\left(h_{1}\right)$ are $-\frac{1}{2}(2 m+1)$. $(a-2) \pm \frac{1}{2}$. Therefore the maximum multiplicity occurs for the roots $\lambda-\frac{1}{2}\{(2 m+1)(a-2) \pm 1\} \alpha_{1}=-(2 m+1) \alpha_{0}-\frac{1}{2}\{(2 m+1) a \pm 1\} \alpha_{1}$

Also by part (b) of Proposition 5.1, we obtain the corresponding symmetries for the root multiplicities. That is,

(1) $\operatorname{mult}\left(n, \frac{1}{2} n a+j\right)=\operatorname{mult}\left(n, \frac{1}{2} n a-j\right)$,

(2) $\operatorname{mult}(2 m, m a+j)=\operatorname{mult}(2 m, m a-j)$,

(3) $\operatorname{mult}\left(2 m+1, \frac{1}{2}\{(2 m+1) a+1\}+j\right)$ $=\operatorname{mult}\left(2 m+1, \frac{1}{2}\{(2 m+1) a-1\}-j\right)$.

EXAmple 5.2 Let $a=3, n=2 m+1=5$. Then the maximum multiplicity occurs for the roots $-5 \alpha_{0}-\frac{1}{2}\{5 \cdot 3 \pm 1\} \alpha_{1}$, which are $-5 \alpha_{0}-8 \alpha_{1}$ and $-5 \alpha_{0}$ $-7 \alpha_{1}$. This can be seen in Table 1 where the corresponding values of $j$ are $j=2$ and $j=3$. The column of the table is symmetric about these maxima.

Another view of the column symmetry is provided by considering directly the action of the Weyl group. Let $r_{i}, i=0,1$ be the simple reflections defined by $r_{i}(\alpha)=\alpha-\alpha\left(h_{i}\right) \alpha_{i}$ where $\alpha_{j}\left(h_{i}\right)=a_{i j}$. Let $\alpha=k_{0} \alpha_{0}+k_{1} \alpha_{1}$ in $\mathrm{g}(a)$, for some fixed $a$. Then

$$
r_{1}(\alpha)=\alpha-\left(2 k_{1}-a k_{0}\right) \alpha_{1}
$$


In particular, for $\alpha=-n \alpha_{0}-(n+j) \alpha_{1}$, as the roots are arranged in Tables $1-4$, we have

$$
\begin{aligned}
r_{1}(\alpha) & =\alpha-(2(-n-j)-a(-n)) \alpha_{1} \\
& =-n \alpha_{0}-((a-1) n-j) \alpha_{1} .
\end{aligned}
$$

Hence $\operatorname{mult}(n, n+j)=\operatorname{mult}(n,(a-1) n-j)$.

For example, for $a=3, n=5$, we have

$$
\operatorname{mult}(5,5+j)=\operatorname{mult}(5,10-j)=\operatorname{mult}(5,5+(5-j)) .
$$

as can be seen in Table 1 .

Proposition 5.1 gives us that $p-q=\lambda(h)$, but does not specify the integers themselves. In order to determine the actual values of $p$ and $q$, and thus the length of the root-chain, we consider root lengths.

Let $\alpha=-n \alpha_{0}-(n+j) \alpha_{1}$ be a root in $g=g(a)$, for some fixed $a \geq 3$. Then

$$
\begin{aligned}
\frac{(\alpha, \alpha)}{2} & =n^{2}+(n+j)^{2}-a n(n+j) \\
& =(2-a) n(n+j)+j^{2}
\end{aligned}
$$

As discussed in Section 4, Moody ([M]) showed that for hyperbolic Kac-Moody algebras $\alpha$ is an imaginary root if and only if $\frac{(\alpha, \alpha)}{2} \leq 0$, and, for $\mathfrak{g}(a), \alpha$ is a real root if and only if $\frac{(\alpha, \alpha)}{2}=1$.

We may suppose without loss of generality, that $j \geq 0$. Then $\alpha$ is an imaginary root (and exists, i.e. has non-zero multiplicity) if

$$
j \leq n(\gamma-1) \text {, where } \gamma=\frac{a+\sqrt{a^{2}-4}}{2} \text { (as in Section 2). }
$$

We also have that $\alpha$ is a real root if and only if

$$
\frac{(\alpha, \alpha)}{2}=(2-a) n(n+j)+j^{2}=1
$$

This is equivalent to the condition that 


$$
j=n\left[\frac{(a-2)+\sqrt{\left(a^{2}-4\right)+\frac{4}{n^{2}}}}{2}\right] \in \mathbf{Z} .
$$

If the right-hand side is integral, then there is a real root (with multiplcity 1 ) $-n \alpha_{0}-(n+j) \alpha_{1}$, for $j=\lfloor n(\gamma-1)\rfloor+1$ (where $\lfloor x\rfloor$ indicates the greatest integer less than or equal to $x)$ and so $p=\lfloor n(\gamma-1)\rfloor+1$.

If the right-hand side of (5.6) is not integral, then the last root in the chain is imaginary and $p=\lfloor n(\gamma-1)\rfloor$.

Example 5.3 Fix $a=3$. Then $\gamma=\frac{3+\sqrt{5}}{2}$, and the right hand side of (5.6) becomes $n\left[\frac{1+\sqrt{5+\frac{4}{n^{2}}}}{2}\right]$. Thus, for $n=3$,

$$
3\left[\frac{1+\sqrt{5+\frac{4}{9}}}{2}\right]=5 \in \mathbf{Z},
$$

and there is a (real) root with $j=\left\lfloor 3\left(\frac{1+\sqrt{5}}{2}\right)\right\rfloor+1=5$. That is, $\alpha=-3 \alpha_{0}$ $8 \alpha_{1}$ is a real root of $\mathrm{g}(3)$. For $n=5$,

$$
5\left[\frac{1+\sqrt{5+\frac{4}{25}}}{2}\right] \notin \mathbf{Z},
$$

and so the last root has $j=\lfloor n(\gamma-1)\rfloor=\left\lfloor 5\left(\frac{1+\sqrt{5}}{2}\right)\right\rfloor=8$. That is, $\alpha=$ $-5 \alpha_{0}-13 \alpha_{1}$ is a root, but $-5 \alpha_{0}-14 \alpha_{1}$ is not.

\section{\$5.2. Multiplicity monotonicity}

As consequence of the symmetry displayed above, we show that, as roots get "larger," their multiplicity increases. More precisely, for the roots $\alpha=$ $(m, n)$ and $\beta=(k, l)$, we define $\alpha \leq \beta$ if and only if $m \leq k$ and $n \leq l$. Then, in the fundamental chamber, we have that if $\alpha \leq \beta$, then mult $\alpha \leq$ mult $\beta$. In general, we have $\operatorname{mult}(m, n) \leq \operatorname{mult}(m+1, n+1)$.

As in the previous subsection,we fix $a \geq 3$, and consider the roots of the algebra $\mathfrak{g}(a)$. Making the identification of the point $(m, n)$ with the root $m \alpha_{0}+$ 


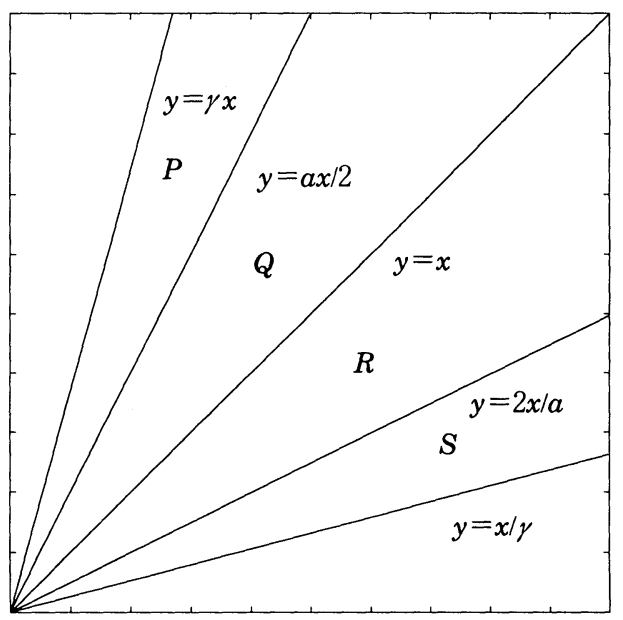

Fig 1.

$n \alpha_{1}$, from Section 4 we have that the imaginary roots of $g(a)$ are the integral points inside the cone $P \cup Q \cup R \cup S$ of Fig 1. (See also Tables 8 and 9 for examples.)

Further, the analysis of the previous subsection showed that the root multiplicities on a vertical line (that is, for a fixed $m$ ) are symmetric about the line $y=\frac{a}{2}$ $x$, increasing monotonically as they approach this line. By symmetry of the root system, the root multiplicities in the horizontal direction are therefore monotonic and symmetric about the line $y=\frac{2}{a} x$. A consequence of this observation is that, if $\alpha=(m, n)$ is a root in the fundamental chamber $Q \cup R$, then any root in the region defined by the intersection of $Q \cup R, x \geq m$ and $y \geq n$, has multiplicity at least equal to that of $\alpha$. Thus we have

Proposition 5.4. Suppose $\alpha, \beta$ are in $Q \cup R$, with $\alpha \leq \beta$. Then mult $\alpha \leq$ mult $\beta$.

Notice that Proposition 5.4 implies that if $\alpha=\left(n_{1}, k n_{1}\right)$ and $\beta=\left(n_{2}, k n_{2}\right)$ with $n_{2}>n_{1}$, (i.e., $\alpha$ and $\beta$ both lie on the line $y=k x$ ), and $\frac{2}{a} \leq k \leq \frac{a}{2}$, then mult $\alpha \leq$ mult $\beta$. The Weyl reflection $r_{1}$ rotates (integral points on) a line $y=m x$ to (integral points on) a line $y=(a-m) x$. In particular, if $\frac{1}{\gamma} \leq k \leq \frac{2}{a}$, so that a 
root $\alpha=(n, k n) \in S$, then $r_{1} \alpha \in Q \cup R$. The remaining case is similar, and we obtain

Corollary 5.5 Let $\alpha=\left(n_{1}, k n_{1}\right), \beta=\left(n_{2}, k n_{2}\right)$ with $n_{2}>n_{1}$. Then mult $\alpha$ $\leq$ mult $\beta$.

This observation was pointed out to us by V. Kac.

Now we may prove our general result.

Proposition 5.6. If $\alpha=(m, n)$ and $\beta=(m+1, n+1)$ are roots of $g(a)$, $a \geq 3$, then mult $\alpha \leq$ mult $\beta$.

Proof. We may suppose without loss of generality than $m \geq n$. From above, if $m>n \gamma$, we must that $m=\lfloor n \gamma\rfloor+1$ and $\alpha$ is real root. Thus $\alpha$ has multiplicity 1 and mult $\beta \geq$ mult $\alpha=1$. From now on we will suppose that $\alpha$ (and hence $\beta$ ) is an imaginary root.

If $\alpha \in Q \cup R$, then $\beta \in Q \cup R$, and by Proposition 5.4 we are done. Hence, we may suppose $\alpha \notin Q \cup R$. By the hypothesis that $m \geq n, \alpha \in S$.

Consider the Weyl reflection $r_{1}$ and the root permutation $\sigma$ defined for $\alpha=$ $(m, n)$ by:

$$
\begin{aligned}
& r_{1}(\alpha)=(m, a m-n), \\
& \sigma(\alpha)=(n, m) .
\end{aligned}
$$

Both $\sigma$ and $r_{1}$ preserve the root multiplicities. Geometrically, $\sigma$ is the reflection in the line $y=x$, and $r_{1}$ reflects a root vertically about the line $y=\frac{a}{2} x$.

Define the following sequences:

$$
\begin{aligned}
& \alpha_{0}=\alpha, \beta_{0}=\beta, \\
& \alpha_{j}=\left(r_{1} \sigma\right)^{j} \alpha, \quad \beta_{j}=\left(r_{1} \sigma\right)^{j} \beta \quad(j \geq 1) .
\end{aligned}
$$

Recall the sequence $\left\{B_{n}\right\}$ from (4.2) and introduce the sequence $\left\{C_{n}\right\}$ defined by:

$$
\begin{array}{ll}
B_{0}=0, & B_{1}=1, \quad B_{n+2}=a B_{n+1}-B_{n} \quad(n \geq 0), \\
C_{0}=1, & C_{1}=1, \quad C_{n+2}=a C_{n+1}-C_{n}(n \geq 0) .
\end{array}
$$


Then

$$
\begin{aligned}
& \alpha_{j}=\left(B_{j} n-B_{j-1} m, B_{j+1} n-B_{j} m\right), \\
& \beta_{j}=\left(B_{j} n-B_{j-1} m+C_{j}, B_{j+1} n-B_{j} m+C_{j+1}\right), \quad j \geq 1 .
\end{aligned}
$$

Note that both $\left\{B_{n}\right\}$ and $\left\{C_{n}\right\}$ are positive and increasing for $a \geq 3$. Hence, $\alpha_{j}$ $\leq \beta_{j}$ for all $j$ (and so $\sigma \alpha_{j} \leq \sigma \beta_{j}$ ).

Let $\eta=(p, q) \in S$, and consider $r_{1} \sigma \eta=(q, a q-p)$. Since $\eta \in S, q \leq \frac{2}{a} p$. Hence $\frac{a}{2} q \leq p, a q-\frac{a}{2} q \leq p$ and $a q-p \leq \frac{a}{2} q$. That is, $r_{1} \sigma \eta \in Q \cup R \cup S$. In fact, if $q>\frac{1}{a-1} p$, then $a q-p>q$ and $r_{1} \sigma \eta \in Q$, and, if $\frac{1}{a-1} p \leq q$ $\leq \frac{a}{a^{2}-2} p$, then $a q-p \geq \frac{2}{a} q$ and $r_{1} \sigma \eta \in R$.

Let $s$ be the smallest non-negative integer such that $\beta_{s} \in Q \cup R$. Note that, although $\alpha_{0} \in S$, it is possible that $\beta_{0} \in R$. Then, for $j<s$, we have $\beta_{j} \in S$.

\section{CLAIm. If $\beta_{j} \in S$, then $\alpha_{j} \in S$.}

Proof. Geometrically, $\beta_{j}=\alpha_{j}+\left(C_{j}, C_{j+1}\right)$. Thus the slope of the line joining $\alpha_{j}$ to $\beta_{j}$ (recall $\alpha_{j} \leq \beta_{j}$ ) is $\frac{C_{j+1}}{C_{j}}>1>\frac{2}{a}$ for $a \geq 3$. Hence $\beta_{j}$ is closer to the line $y=\frac{2}{a} x$ than $\alpha_{j}$, and, if $\beta_{j} \in S$, then so is $\alpha_{j}$.

If $\alpha_{s} \in Q \cup R$, then we have $\alpha_{s} \leq \beta_{s}, \alpha_{s}, \beta_{s} \in Q \cup R$, and, by Proposition 5.4 , we are done.

Now suppose $\alpha_{s} \notin Q \cup R$. That is, $\alpha_{s} \in S$. Note that, in general, for $\eta=$ $(p, q) \in S, r_{1} \sigma \eta \not \eta \eta$, but $\sigma r_{1} \sigma \eta \leq \eta$, or, equivalently, $r_{1} \sigma \eta \leq \sigma \eta$. To see this, recall that since $r_{1} \sigma \eta=(q, a q-p)$, we have $q \leq p$, but $a q-p$ may be greater than $q$, However, $a q-p \leq p$, since $q \leq \frac{2}{a} p$. Hence, $\left(r_{1} \sigma\right)^{j} \eta \leq \sigma^{j} \eta$.

Let $r$ be the smallest such that $\alpha_{r} \in Q \cup R$. Then

$$
\alpha_{r} \leq\left(r_{1} \sigma\right)^{r-s} \alpha_{s} \leq \sigma^{r-s} \alpha_{s} \leq \sigma^{r-s} \beta_{s} .
$$

That is, either $\alpha_{r} \leq \beta_{s}$, or $\sigma \alpha_{r} \leq \beta_{s}$. In either case, we have mult $\alpha \leq$ mult $\beta$. 
COROLlary 5.7. If $\alpha=(m, n)$ and $\beta=(m+j, n+j)$ are roots of $\mathfrak{g}(a), a \geq$ 3 with $j$ a non-negative integer, then mult $\alpha \leq$ mult $\beta$.

\section{§5.3. Multiplicity questions}

In this final section we raise some questions concerning further possible relationships among the root multiplicities for given algebras.

The analysis of Section 4 revealed a connection between the root multiplicity and the root length. Specifically, we showed that an imaginary root of $\mathrm{g}(a)$ of length $-2 k$ lies on the hyperbola $\mathscr{H}_{k}$ and is $W$-conjugate to some root in $\Omega_{k}$. If $\left|\Omega_{k}\right|=1$, all roots of length $-2 k$ must have the same multiplicity. If $\left|\Omega_{k}\right|>1$, this need not be the case and there may be roots of the same length, but with different multiplicities. For example, the roots $(9,17)$ and $(11,11)$ of $\mathfrak{g}(4)$ both have length -484 , but have multiplicities 18900 and 18901 , respectively.

A natural question is whether relationship between length and multiplicity is monotonic. That is, does the multiplicity always increase as (the magnitude of) the length increases? Geometrically, this would imply that the multiplicity of any root "inside" a hyperbola is greater than or equal to the multiplicity of any root on the hyperbola. In general, this is not true. We do not know of any counterexamples for the algebras $\mathrm{g}(3)$ and $\mathfrak{g}(4)$, but in $\mathfrak{g}(5)$, the root $\alpha=(3,7)$ has length $-2 k=$ -94 and multiplicity 9 , while the $\operatorname{root} \beta=(4,4)$ has length -96 and multiplicity 8. There are similar examples for $a=6$ and 7 . In view of the apparent exponential increase in multiplicity with respect to length, it would be very interesting to discover precisely the conditions required for this to happen.

Note for comparison the mysterious formulas of $[\mathrm{FF}]$ and $[\mathrm{FFR}]$ for $H A_{1}^{(1)}$, [KMW] for $E_{10}$, and $[\mathrm{KM}]$ for $H A_{n}^{(1)}$, which showed that, for roots of low level in certain hyperbolic Kac-Moody algebras, the multiplicity depends only on the length of the root and increases monotonically with the root length.

In the previous section, we showed that root multiplicities increase monotonically along the lines $y=x+j, j \in \mathbf{Z}, j \geq 0$. It is also interesting to consider the lines perpendicular to these, the integral points of which represent roots of a given height $t$. It can easily be seen that, for the roots in the tables, the multiplicities increase monotonically to maximum at $(m, m)$ for $t$ even, and $(m-1$, $m+1)$ for $t$ odd. We believe that this may be true in general. That is, we have 
the following conjecture:

CONJECTURE 5.8. Let $m, n$ be non-negative integers with $m \leq n-2$. Then, for any $g(a), a \geq 3$, mult $(m, n) \leq \operatorname{mult}(m+1, n-1)$.

\section{Root multiplicity tables}

In this section we present some tables of root multiplicities of the rank 2 Kac-Moody algebras $\mathfrak{g}(a)$ to illustrate the relationships explained in the text.

In Tables 1-7, we consider the roots of the form $n \alpha_{0}+(n+j) \alpha_{1}$ in various different settings. First, in Tables $1-4$, we consider the root multiplicities in a given algebra as $n$ and $j$ vary. That is, we have a fixed $a=3, \ldots, 6$ in each table. For comparison, Table 5 then gives the root multiplicities for the same roots in the free Lie algebra of rank 2. Tables 6 and 7 rearrange the roots to illustrate the stability theorem of Section 3 . Here we fix $j=0$ and $j=1$, respectively, and allow $a$ and $n$ to vary. The stability theorem and the precise relationships indicated in (2.24) and (2.25) can be seen clearly.

Tables 8 and 9 present a different view of the algebras. In these tables we give the multiplicities for roots written in the form $m \alpha_{0}+n \alpha_{0}$ for $\mathrm{g}(a), a=3$ and 4 , with the regions $P, Q, R, S$ from Fig 1 , and some of the hyperbolas $\mathscr{H}_{k}$ superimposed. The reader will easily be able to see Proposition 5.6 and the force of Conjecture 5.8 .

The root multiplicities were calculated using the Kacmoody algorithm of A.J. Coleman of Queen's University. The program itself was written by R. McCann and I. Wilmott, and modified by R. McCann and M. Roth. The algorithm is based upon Berman-Moody's formula (1.14). 
Root multiplicities for $a=3$

\begin{tabular}{|l|l|l|l|l|l|l|l|l|l|l|}
\hline$j \backslash n$ & 1 & 2 & 3 & 4 & 5 & 6 & 7 & 8 & 9 & 10 \\
\hline 0 & 1 & 1 & 3 & 6 & 16 & 39 & 107 & 288 & 808 & 2278 \\
1 & 1 & 2 & 4 & 9 & 23 & 60 & 162 & 449 & 1267 & 3630 \\
2 & 1 & 1 & 4 & 9 & 27 & 73 & 211 & 600 & 1754 & 5130 \\
3 & 0 & 1 & 3 & 9 & 27 & 80 & 240 & 720 & 2167 & 6555 \\
4 & 0 & 0 & 2 & 6 & 23 & 73 & 240 & 758 & 2407 & 7554 \\
5 & 0 & 0 & 1 & 4 & 16 & 60 & 211 & 720 & 2407 & 7936 \\
6 & 0 & 0 & 0 & 1 & 9 & 39 & 162 & 600 & 2167 & 7554 \\
7 & 0 & 0 & 0 & 0 & 4 & 23 & 107 & 449 & 1754 & 6555 \\
8 & 0 & 0 & 0 & 0 & 1 & 9 & 60 & 288 & 1267 & 5130 \\
9 & 0 & 0 & 0 & 0 & 0 & 3 & 27 & 162 & 808 & 3630 \\
10 & 0 & 0 & 0 & 0 & 0 & 0 & 9 & 73 & 449 & 2278 \\
\hline
\end{tabular}

Table 1

Root multiplicities for $a=4$

\begin{tabular}{|l|l|l|l|l|l|l|l|l|l|l|}
\hline$j \backslash n$ & 1 & 2 & 3 & 4 & 5 & 6 & 7 & 8 & 9 & 10 \\
\hline 0 & 1 & 1 & 3 & 8 & 23 & 64 & 195 & 590 & 1850 & 5861 \\
1 & 1 & 2 & 5 & 13 & 36 & 106 & 321 & 995 & 3144 & 10088 \\
2 & 1 & 2 & 6 & 16 & 50 & 151 & 480 & 1521 & 4928 & 16070 \\
3 & 1 & 2 & 6 & 20 & 63 & 202 & 660 & 2169 & 7185 & 23990 \\
4 & 0 & 1 & 6 & 20 & 72 & 243 & 840 & 2860 & 9810 & 33605 \\
5 & 0 & 1 & 5 & 20 & 75 & 276 & 995 & 3550 & 12590 & 44498 \\
6 & 0 & 0 & 3 & 16 & 72 & 283 & 1100 & 4115 & 15238 & 55717 \\
7 & 0 & 0 & 2 & 13 & 63 & 276 & 1137 & 4510 & 17441 & 66284 \\
8 & 0 & 0 & 1 & 8 & 50 & 243 & 1100 & 4635 & 18900 & 74886 \\
9 & 0 & 0 & 0 & 5 & 36 & 202 & 995 & 4510 & 19409 & 80600 \\
10 & 0 & 0 & 0 & 2 & 23 & 151 & 840 & 4115 & 18900 & 82543 \\
\hline
\end{tabular}

Table 2 
Root multiplicities for $a=5$

\begin{tabular}{|l|l|l|l|l|l|l|l|l|l|l|}
\hline$j \backslash n$ & 1 & 2 & 3 & 4 & 5 & 6 & 7 & 8 & 9 & 10 \\
\hline 0 & 1 & 1 & 3 & 8 & 25 & 73 & 232 & 734 & 2400 & 7935 \\
1 & 1 & 2 & 5 & 14 & 41 & 125 & 395 & 1277 & 4207 & 14073 \\
2 & 1 & 2 & 7 & 19 & 61 & 190 & 625 & 2059 & 6930 & 23511 \\
3 & 1 & 3 & 8 & 26 & 84 & 276 & 928 & 3150 & 10800 & 37361 \\
4 & 1 & 2 & 9 & 30 & 108 & 370 & 1300 & 4540 & 16005 & 56536 \\
5 & 0 & 2 & 9 & 35 & 130 & 475 & 1725 & 6250 & 22628 & 81950 \\
6 & 0 & 1 & 8 & 35 & 147 & 566 & 2175 & 8177 & 30612 & 113869 \\
7 & 0 & 1 & 7 & 35 & 156 & 650 & 2612 & 10262 & 39725 & 152243 \\
8 & 0 & 0 & 5 & 30 & 156 & 698 & 2993 & 12300 & 49525 & 195928 \\
9 & 0 & 0 & 3 & 26 & 147 & 720 & 3275 & 14175 & 59400 & 243339 \\
10 & 0 & 0 & 2 & 19 & 130 & 698 & 3425 & 15645 & 68625 & 291685 \\
\hline
\end{tabular}

Table 3

Root multiplicities for $a=6$

\begin{tabular}{|l|l|l|l|l|l|l|l|l|l|l|}
\hline$j \backslash n$ & 1 & 2 & 3 & 4 & 5 & 6 & 7 & 8 & 9 & 10 \\
\hline 0 & 1 & 1 & 3 & 8 & 25 & 75 & 243 & 785 & 2616 & 8815 \\
1 & 1 & 2 & 5 & 14 & 42 & 131 & 421 & 1387 & 4654 & 15855 \\
2 & 1 & 2 & 7 & 20 & 65 & 206 & 686 & 2297 & 7857 & 27111 \\
3 & 1 & 3 & 9 & 29 & 94 & 312 & 1059 & 3641 & 12660 & 44450 \\
4 & 1 & 3 & 11 & 36 & 128 & 441 & 1557 & 5489 & 19558 & 69943 \\
5 & 1 & 3 & 12 & 45 & 165 & 602 & 2189 & 7967 & 29050 & 106162 \\
6 & 0 & 2 & 12 & 50 & 203 & 774 & 2951 & 11085 & 41594 & 155545 \\
7 & 0 & 2 & 12 & 56 & 238 & 966 & 3283 & 14904 & 57540 & 220728 \\
8 & 0 & 1 & 11 & 56 & 266 & 1143 & 4769 & 19305 & 77028 & 303534 \\
9 & 0 & 1 & 9 & 56 & 284 & 1311 & 5737 & 24228 & 99932 & 405426 \\
10 & 0 & 0 & 7 & 50 & 290 & 1436 & 6664 & 29389 & 125804 & 526177 \\
\hline
\end{tabular}

Table 4 
Root multiplicities for free Lie algebra of rank 2

\begin{tabular}{|l|l|l|l|l|l|l|l|l|l|l|}
\hline$j \backslash n$ & 1 & 2 & 3 & 4 & 5 & 6 & 7 & 8 & 9 & 10 \\
\hline 0 & 1 & 1 & 3 & 8 & 25 & 75 & 245 & 800 & 2700 & 9225 \\
1 & 1 & 2 & 5 & 14 & 42 & 132 & 429 & 1430 & 4862 & 16796 \\
2 & 1 & 2 & 7 & 20 & 66 & 212 & 715 & 2424 & 8398 & 29372 \\
3 & 1 & 3 & 9 & 30 & 99 & 333 & 1144 & 3978 & 13995 & 49742 \\
4 & 1 & 3 & 12 & 40 & 143 & 497 & 1768 & 6288 & 22610 & 81686 \\
5 & 1 & 4 & 15 & 55 & 200 & 728 & 2652 & 9690 & 35530 & 130750 \\
6 & 1 & 4 & 18 & 70 & 273 & 1026 & 3876 & 14520 & 54477 & 204248 \\
7 & 1 & 5 & 22 & 91 & 364 & 1428 & 5537 & 21318 & 81719 & 312455 \\
8 & 1 & 5 & 26 & 112 & 476 & 1932 & 7752 & 30624 & 120175 & 468611 \\
9 & 1 & 6 & 30 & 140 & 612 & 2583 & 10659 & 43263 & 173583 & 690690 \\
10 & 1 & 6 & 35 & 168 & 775 & 3384 & 14421 & 60060 & 246675 & 1001400 \\
\hline
\end{tabular}

Table 5

Root multiplicities of $\mathfrak{g}(a)$, for $j=0$

\begin{tabular}{|l|l|l|l|l|l|l|l|l|l|l|}
\hline$a \backslash n$ & 1 & 2 & 3 & 4 & 5 & 6 & 7 & 8 & 9 & 10 \\
\hline 3 & 1 & 1 & 3 & 6 & 16 & 39 & 107 & 288 & 808 & 2278 \\
4 & 1 & 1 & 3 & 8 & 23 & 64 & 195 & 590 & 1850 & 5861 \\
5 & 1 & 1 & 3 & 8 & 25 & 73 & 232 & 734 & 2400 & 7935 \\
6 & 1 & 1 & 3 & 8 & 25 & 75 & 243 & 785 & 2616 & 8815 \\
7 & 1 & 1 & 3 & 8 & 25 & 75 & 245 & 798 & 2683 & 9121 \\
8 & 1 & 1 & 3 & 8 & 25 & 75 & 245 & 800 & 2698 & 9206 \\
9 & 1 & 1 & 3 & 8 & 25 & 75 & 245 & 800 & 2700 & 9223 \\
10 & 1 & 1 & 3 & 8 & 25 & 75 & 245 & 800 & 2700 & 9225 \\
\hline
\end{tabular}

Table 6

Root multiplicities of $\mathrm{g}(a)$, for $j=1$

\begin{tabular}{|l|l|l|l|l|l|l|l|l|l|l|}
\hline$a \backslash n$ & 1 & 2 & 3 & 4 & 5 & 6 & 7 & 8 & 9 & 10 \\
\hline 3 & 1 & 2 & 4 & 9 & 23 & 60 & 162 & 449 & 1267 & 3630 \\
4 & 1 & 2 & 5 & 13 & 36 & 106 & 321 & 995 & 3144 & 10088 \\
5 & 1 & 2 & 5 & 14 & 41 & 125 & 395 & 1277 & 4207 & 14073 \\
6 & 1 & 2 & 5 & 14 & 42 & 131 & 421 & 1387 & 4654 & 15855 \\
7 & 1 & 2 & 5 & 14 & 42 & 132 & 428 & 1421 & 4809 & 16522 \\
8 & 1 & 2 & 5 & 14 & 42 & 132 & 429 & 1429 & 4852 & 16732 \\
9 & 1 & 2 & 5 & 14 & 42 & 132 & 429 & 1430 & 4861 & 16785 \\
10 & 1 & 2 & 5 & 14 & 42 & 132 & 429 & 1430 & 4862 & 16795 \\
\hline
\end{tabular}

Table 7 


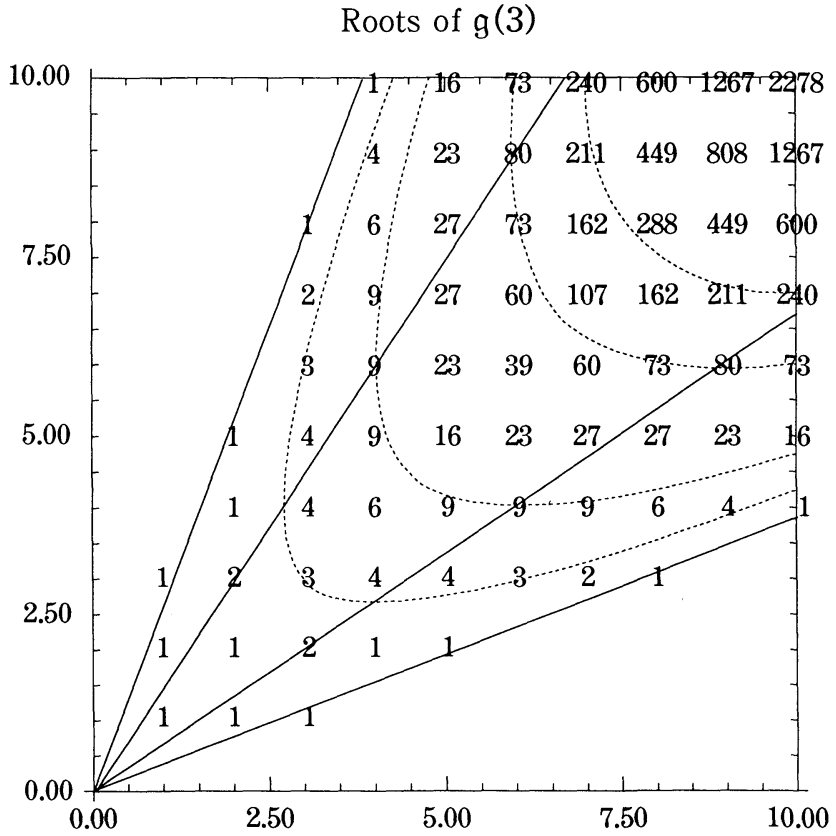

Table 8

Roots of $g(4)$

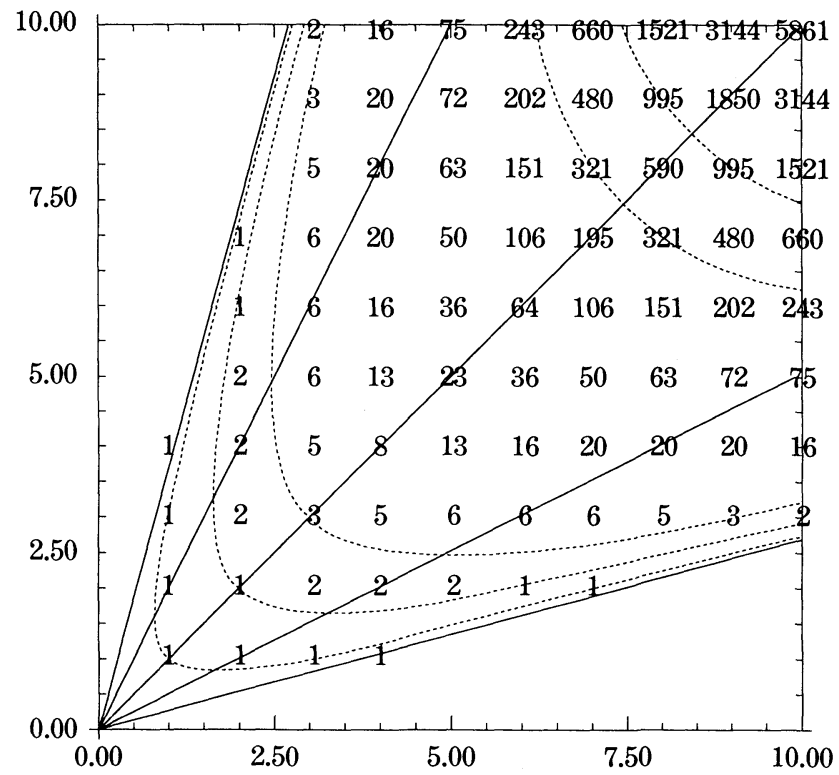

Table 9 


\section{REFERENCES}

[BKM] Benkart, G. M., Kang S.-J., Misra, K. C., Graded Lie algebras of Kac-Moody type, Adv. in Math., 97 (1993), 154-190.

[BM] Berman, S., Moody, R. V., Multiplicities in Lie alebras, Proc. Amer. Math. Soc., 76 (1979), 223-228.

[F] Feingold, A. J., A hyperbolic GCM and the Fibonacci numbers, Proc. Amer. Math. Soc., 80 (1980), 379-385.

[FF] Feingold, A. J., Frenkel, I. B., A hyperbolic Kac-Moody algebra and the theory of Siegel modular forms of genus 2, Math. Ann., 263 (1983), 87-144.

[FFR] Feingold, A. J., Frenkel, I. B., Ries, J. F. X., Representations of hyperbolic Kac-Moody algebras, J. Algebra, 156 (1993), 433-453.

[Fr] Frenkel, I. B., Representations of Kac-Moody algebras and dual resonance models, Applications of Group Theory in Physics and Mathematical Physics, Lectures in Applied Math., Amer. Math. Soc., 21 (1985), 325-353.

[GK] Gabber, O., Kac, V. G., On defining relations of certain infinite dimensional Lie algebras, Bull. Amer. Math. Soc., 5 (1981), 185-189.

[GL] Garland, H., Lepowsky, J., Lie algebra homology and the Macdonald-Kac formulas, Invent. Math., 34 (1976), 37-76.

[K] Kac, V. G., Infinite dimensional Lie algebras, 3rd ed. Cambridge University Press (1990).

[KMW] Kac, V. G., Moody, R. V., Wakimoto, M., On $E_{10}$, Differential Geometrical Methods in Theoretical Physics, Bleuler, K., Werner, M. (eds.), Kluwer Academic Publishers (1988), 109-128.

[Ka1] Kang, S.-J., Kac-Moody Lie algebras, spectral sequences, and the Witt formula, Trans. Amer. Math. Soc., 339 (1993), 463-495.

[Ka2] Kang, S. -J., Root multiplicities of Kac-Moody algebras, Duke Math. J., 74, No. 3 (1994), 635-666.

[KM] Kang, S. -J., Melville, D. J., Root multiplicities of the Kac-Moody algebras $H A_{n}^{(1)}$, J. Algebra. 170 (1994), 277-299.

[LM] Lepowsky, J., Moody, R. V., Hyperbolic Lie algebras and quasi-regular cusps on Hilbert modular sufaces, Math. Ann., 245 (1979) 63-88.

[Li] Liu, L. -S., Kostant's formula for Kac-Moody Lie algebras, J. Algebra, 149 (1992), 155-178.

[M] Moody, R. V., Root systems of hyperbolic type, Adv. in Math., 33 (1979), $144-160$.

[Se] Serre, J. P., Lie Algebras and Lie Groups, 1964 Lectures given at Harvard University, Benjamin, New York (1965).

S. -J. Kang

Department of Mathematics

University of Notre Dame

Notre Dame, IN 46556

U.S.A.

Current Address

Department of Mathematics

College of Natural Sciences

Seoul National University

Seoul 151-742, Korea
D. J. Meyille

Department of Mathematics

St. Lawrence University

Canton, NY 13617

U.S.A. 